\title{
On an Optimal $L^{1}$-Control Problem in Coefficients for Linear Elliptic Variational Inequality
}

\author{
Olha P. Kupenko ${ }^{1,2}$ and Rosanna Manzo ${ }^{3}$ \\ ${ }^{1}$ Department of System Analysis and Control, National Mining University, Karl Marx Avenue 19, Dnipropetrovsk 49005, Ukraine \\ ${ }^{2}$ The Research Laboratory for Nonlinear Analysis of Differential-Operator Systems of Institute for Applied and System Analysis in \\ National Technical University of Ukraine "Kiev Polytechnical Institute", Peremogy Avenue 37, building 35, Kiev 03056, Ukraine \\ ${ }^{3}$ Dipartimento di Ingegneria dell'Informazione, Ingegneria Elettrica e Matematica Applicata, Università degli Studi di Salerno, \\ Via Giovanni Paolo II 132, 84084 Fisciano, Italy
}

Correspondence should be addressed to Rosanna Manzo; rmanzo@unisa.it

Received 27 January 2013; Accepted 24 June 2013

Academic Editor: Matti Lassas

Copyright (C) 2013 O. P. Kupenko and R. Manzo. This is an open access article distributed under the Creative Commons Attribution License, which permits unrestricted use, distribution, and reproduction in any medium, provided the original work is properly cited.

We consider optimal control problems for linear degenerate elliptic variational inequalities with homogeneous Dirichlet boundary conditions. We take the matrix-valued coefficients $A(x)$ in the main part of the elliptic operator as controls in $L^{1}\left(\Omega ; \mathbb{R}^{N(N+1) / 2}\right)$. Since the eigenvalues of such matrices may vanish and be unbounded in $\Omega$, it leads to the "noncoercivity trouble." Using the concept of convergence in variable spaces and following the direct method in the calculus of variations, we establish the solvability of the optimal control problem in the class of the so-called $\mathrm{H}$-admissible solutions.

\section{Introduction}

The aim of this work is to study the existence of optimal controls in the matrix-valued coefficients associated with a linear degenerate elliptic variational inequality with homogeneous Dirichlet boundary conditions. The controls are taken as the matrix $A \in L^{1}\left(\Omega ; \mathbb{R}^{N(N+1) / 2}\right)$ of the coefficients in the main part of the elliptic operator. The most important feature of such controls is the fact that the eigenvalues of the matrix A may either vanish on subsets with zero Lebesgue measure or be unbounded. In this case, the precise answer for the question of existence or nonexistence of optimal solutions heavily depends on the class of chosen admissible controls. Using the direct method in the calculus of variations, we discuss the solvability of this optimal control problem in the class of the so-called $H$-admissible solutions (see, for instance, $[1,2])$. It should be emphasized that in contrast to the paper [3], we do not make use of any relaxations for the original optimal control problem. We note that there are many physical phenomena related to the mathematical theory of cloaking, design of advanced materials, behaviour of the optical meta-materials in the context of cloaking, and others which lead to the appearance of variational inequalities and matrices in the main part of elliptic operators with degenerate spectrum (see, for instance, [4-8]). In particular, the mathematical models of equilibrium of continuous media which are "perfect" insulators or "perfect" conductors (see [9]) need eigenvalues of the matrix $A$ either to vanish somewhere or to be unbounded.

Also similar matrices with degenerate eigenvalues arise in an intensively studied nowadays area of complex dynamical networks [10-12]. Namely, such matrices describe an inner configuration of networks; that is, they can be interpreted as connection matrices among nodes $[10,11]$. In fact, many properties of complex networks are mainly determined by their configuration matrices, in particular, such as network synchronizability as well as robustness and fragility of a network in synchronization. This fact could be a good motivation to work out techniques for achieving synchronization for dynamical networks which cannot synchronize, taking configuration matrices as controls. Such situation would be similar to the one described in the present paper, when varying a chosen control leads to changing a control object's structure. Hence, one of the main goals of the paper is 
to construct a mathematical framework for dealing with problems of this kind.

It is worth noticing that even though numerous articles (see, for instance, [13-18] and references therein) are devoted to variational and nonvariational approaches to problems related to linear and nonlinear elliptic systems, only few deal with optimal control problems for degenerate partial differential equations and variational inequalities (see e.g., $[1,2,19-23])$.

In this paper, we deal with an optimal control problem in coefficients for the following linear degenerate elliptic variational inequality:

$$
\begin{gathered}
\sum_{i, j=1}^{N} \int_{\Omega}\left(a_{i j}(x) \frac{\partial y}{\partial x_{j}}\right)\left(\frac{\partial v}{\partial x_{i}}-\frac{\partial y}{\partial x_{i}}\right) d x+\int_{\Omega} y(v-y) d x \\
\geq \int_{\Omega} f(v-y) d x \quad \forall v \in K_{A} .
\end{gathered}
$$

Here, $f \in L^{2}(\Omega)$ is a given distribution, $K_{A}$ is a convex closed subset of the space $W_{0}^{1,1}(\Omega)$, and $A$ is a measurable nonnegative square symmetric matrix on a bounded open domain $\Omega \subset \mathbb{R}^{N}(N \geq 2)$ such that $A \in B V\left(\Omega ; \mathbb{R}^{N(N+1) / 2}\right)$.

We furnish this control object by the following cost functional:

$$
\begin{aligned}
I(A, y)= & \int_{\Omega}\left|y(x)-y_{d}(x)\right|^{2} d x \\
& +\int_{\Omega}(\nabla y(x), A(x) \nabla y(x))_{\mathbb{R}^{N}} d x+\|A\|_{B V(\Omega)} .
\end{aligned}
$$

The characteristic feature of this optimal control problem is the fact that the eigenvalues of admissible matrices $A$ may vanish and be unbounded on subdomains of $\Omega$ with zero Lebesgue measure. Indeed, we assume that for every admissible matrix $A$ there exist two nonnegative $L^{1}(\Omega)$-functions $\zeta$ and $\beta$ such that $\zeta^{-1} \in L^{1}(\Omega), \zeta^{-1} \notin L^{\infty}(\Omega)$, and

$$
\begin{array}{r}
\zeta(x)\|\xi\|_{\mathbb{R}^{N}}^{2} \leq(A(x) \xi, \xi)_{\mathbb{R}^{N}} \leq \beta(x)\|\xi\|_{\mathbb{R}^{N}}^{2} \\
\text { a.e. in } \Omega \quad \forall \xi \in \mathbb{R}^{N} .
\end{array}
$$

Because of this, such matrices are sometimes referred to as matrices with degenerate spectrum. It is clear that conditions (3) lead us to the so-called "noncoercivity trouble." It means that boundary value problem (1) for some locally integrable matrix-valued functions $A$ may exhibit the Lavrentieff phenomenon, the nonuniqueness of weak solutions, as well as other surprising consequences. So, in general, the mapping $A \mapsto y(A)$ can be multivalued. On the other hand, this problem is ill-posed, in general. It means that there are no reasons to suppose that for every $f \in L^{2}(\Omega)$ and $A \in$ $L^{1}\left(\Omega ; \mathbb{R}^{N(N+1) / 2}\right)$, the problem (1) admits at least one weak solution in $H_{0}^{1}(\Omega)$. Thus, it makes it impossible to apply classical theorems (see $[24,25]$ ) to establish solvability of the corresponding variational inequality and, hence, the regularity of the optimal control problem associated with it.
Besides, we show that for every admissible control function $A$ it is plausible to consider weak solutions of problem (1) as elements of the corresponding weighted Sobolev spaces $W(\Omega, A d x)$ which are constructed as sets of functions $y \in W_{0}^{1,1}(\Omega)$ for which the norm

$$
\|y\|_{A}=\left(\int_{\Omega}\left(y^{2}+(\nabla y, A(x) \nabla y)_{\mathbb{R}^{N}}\right) d x\right)^{1 / 2}
$$

is finite. In addition, we note that even if the original elliptic operator is nondegenerate, that is, admissible controls $A(x)$ are such that

$$
\alpha\|\xi\|_{\mathbb{R}^{N}}^{2} \leq(\xi, A(x) \xi)_{\mathbb{R}^{N}} \leq \beta\|\xi\|_{\mathbb{R}^{N}}^{2} \quad \xi \in \mathbb{R}^{N}
$$

with $\alpha>0$, the majority of optimal control problems in coefficients have no solution in general (see for instance $[15,26-28])$.

Since for atypical matrices with degenerate spectrum, namely, such that $A, A^{-1} \notin L^{\infty}\left(\Omega ; \mathbb{R}^{N(N+2) / 2}\right)$, the space of smooth compactly supported functions $C_{0}^{\infty}(\Omega)$ is not dense in $W(\Omega ; A d x)$ in general, we discuss the solvability of this optimal control problem in the class of the so-called $H$ admissible solutions. To this end, we consider, as the main solution space, the closure of $C_{0}^{\infty}(\Omega)$ in $W(\Omega ; A d x)$-norm and denote this space by $H(\Omega ; A d x)$. In order to specify the set $K_{A}$ in (1), we assume that there exists a closed convex subset $K$ of $W_{0}^{1,1}(\Omega)$ such that $K \cap C_{0}^{\infty}(\Omega) \neq \emptyset$. In this case, we set $K_{A}$ as the closure of $\{K \cap H(\Omega ; A d x)\}$ with respect to $W(\Omega ; A d x)$-norm (4).

At the same time, having formulated the optimal control problem in coefficients in terms of the corresponding weighted Sobolev spaces, we are facing another kind of challenge: changing the control matrix changes not only the solution space $H(\Omega ; A d x)$ but also the control object itself. In fact, it means that the set of admissible solutions to the previous optimal control problem is a family of pairs, each of which belongs to the correspondent space. Therefore, in this situation there are several possible settings of the variational inequality and optimal control problem associated with it, which depend on the choice of solution space. The main questions are what is the right setting of the optimal control problem with $L^{1}$-controls in coefficients, and what is the right class of admissible solutions to the considered problem? As we show in this paper, the precise answer for the question of existence or nonexistence of optimal solutions heavily depends on the class of chosen admissible controls.

The paper is organized as follows. Section 2 concerns some notation and preliminaries. In Section 3, we describe the main notion of weak and strong convergence in variable $L^{2}$-spaces. Section 4 gives a collection of auxiliary results that we will use in the sequel. In particular, following Kogut and Leugering [29], we introduce the concept of $w$ convergence in variable space $L^{1}\left(\Omega ; \mathbb{R}^{N(N+1) / 2}\right) \times H(\Omega ; A d x)$ and give sufficient conditions of sequential $w$-compactness. In Section 5, we introduce the admissible control constrains, the class of $H$-optimal admissible solutions, and discuss the regularity of the corresponding optimal control problem. In Section 6, using the direct method in the calculus of 
variations, we prove the existence of the so-called $H$-optimal solutions to the original problem.

\section{Notation and Preliminaries}

Let $\Omega$ be a bounded open subset of $\mathbb{R}^{N}(N \geq 2)$ with Lipschitz boundary. We assume that the boundary of $\Omega$, denoted by $\partial \Omega$, has positive $(N-1)$-dimensional measure.

By $C_{0}^{\infty}(\Omega)$, we denote the set of functions from $C_{0}^{\infty}\left(\mathbb{R}^{N}\right)$, compactly supported in $\Omega$. We define the Banach space $W_{0}^{1,1}(\Omega)$ as the closure of $C_{0}^{\infty}(\Omega)$ in the classical Sobolev space $W^{1,1}(\Omega)$. For any subset $E \subset \Omega$, we denote by $|E|$ its $N$ dimensional Lebesgue measure $\mathscr{L}^{N}(E)$.

Symmetric Matrices with Degenerate Eigenvalues. We denote by $\mathbb{S}^{N}:=\mathbb{R}^{N(N+1) / 2}$ the set of all symmetric matrices $\vec{\xi}=\left[\xi_{i j}\right]_{i, j=1}^{N},\left(\xi_{i j}=\xi_{j i}\right)$. We suppose that $\mathbb{S}^{N}$ is endowed with the Euclidian scalar product $\vec{\xi} \cdot \vec{\eta}=\operatorname{tr}(\vec{\xi} \vec{\eta})$ and with the corresponding Euclidian norm $\|\vec{\xi}\|_{\mathbb{S}^{N}}=(\vec{\xi} \cdot \vec{\xi})^{1 / 2}$. Let $L^{1}(\Omega)^{N(N+1) / 2}=L^{1}\left(\Omega ; \mathbb{S}^{N}\right)$ be the space of integrable functions whose values are symmetric matrices.

Let $\alpha \in \mathbb{R}$ be a fixed positive value. Let $\zeta_{a d}: \Omega \rightarrow \overline{\mathbb{R}}$ be a given function satisfying the properties

$$
\zeta_{a d} \in L^{1}(\Omega), \quad \zeta_{a d}^{-1} \in L^{1}(\Omega), \quad \zeta_{a d}^{-1} \notin L^{\infty}(\Omega) .
$$

Let $\Psi_{*}$ be a nonempty compact subset of $L^{1}(\Omega)$ such that for any $\zeta_{*} \in \Psi_{*}$ the following conditions hold true:

$$
\begin{gathered}
\zeta_{a d}(x)<\zeta_{*}(x) \quad \text { a.e. in } \Omega, \\
\zeta_{*}: \Omega \longrightarrow \mathbb{R}_{+} \quad \text { is a smooth function } \\
\text { along the boundary } \partial \Omega, \\
\zeta_{*}=\alpha \quad \text { on } \partial \Omega .
\end{gathered}
$$

By $\mathfrak{M}_{\alpha}^{\beta}(\Omega)$, we denote the set of all matrices $A(x)=$ $\left[a_{i j}(x)\right] \in L^{1}\left(\Omega ; \mathbb{S}^{N}\right)$ such that

$$
\begin{gathered}
A(x) \leq \beta(x) I \quad \text { a.e. in } \Omega, \\
\exists \zeta_{*} \in \Psi_{*} \quad \text { s.t. } \zeta_{*}(x) I \leq A(x) \quad \text { a.e. in } \Omega .
\end{gathered}
$$

Here, $\beta \in L^{1}(\Omega)$ is a given function such that $\beta(x)>0$ a.e. in $\Omega$ and such that $\beta \cdot \zeta_{\text {ad }}^{-1} \in L^{\infty}(\Omega), I$ is the identity matrix in $\mathbb{R}^{N \times N}$, and (8)-(9) should be considered in the sense of quadratic forms. Therefore, (8)-(9) imply the following inequalities:

$$
\begin{gathered}
\text { if } A \in \mathfrak{M}_{\alpha}^{\beta}(\Omega) \text {, then }\|A(x)\|_{L^{1}\left(\Omega ; \mathbb{S}^{N}\right)} \leq\|\beta\|_{L^{1}(\Omega)}<+\infty \text {, } \\
\zeta_{*}(x)\|\xi\|_{\mathbb{R}^{N}}^{2} \leq(A(x) \xi, \xi)_{\mathbb{R}^{N}} \leq \beta(x)\|\xi\|_{\mathbb{R}^{N}}^{2} \\
\text { a.e. in } \Omega \quad \forall \xi \in \mathbb{R}^{N} .
\end{gathered}
$$

Remark 1. Since every measurable matrix-valued function $A: \Omega \rightarrow \mathbb{S}^{N}$ can be associated with the collection of its eigenvalues $\left\{\lambda_{1}^{A}, \ldots, \lambda_{N}^{A}\right\}$, where each $\lambda_{k}^{A}=\lambda_{k}^{A}(x)$ is counted with its multiplicity, (9), in view of the properties of the class $\Psi_{*}$, means that eigenvalues of matrices $A \epsilon$ $\mathfrak{M}_{\alpha}^{\beta}(\Omega)$ may vanish and be unbounded on subdomains of $\Omega$ with zero Lebesgue measure. Because of this, these matrices are sometime referred to as matrices with degenerate spectrum.

Weighted Sobolev Spaces. To each matrix $A \in \mathfrak{M}_{\alpha}^{\beta}(\Omega)$, we will associate two weighted Sobolev spaces:

$$
W_{A}=W(\Omega ; A d x), \quad H_{A}=H(\Omega ; A d x),
$$

where $W_{A}$ is the set of functions $y \in W_{0}^{1,1}(\Omega)$ for which the norm, given by (4), is finite, and $H_{A}$ is the closure of $C_{0}^{\infty}(\Omega)$ in $W_{A}$. Note that due to inequality (11) and estimates

$$
\begin{gathered}
\int_{\Omega}|y| d x \leq\left(\int_{\Omega}|y|^{2} d x\right)^{1 / 2}|\Omega|^{1 / 2} \leq C\|y\|_{A^{\prime}} \\
\int_{\Omega}\|\nabla y\|_{\mathbb{R}^{N}} d x \\
\leq\left(\int_{\Omega}\|\nabla y\|_{\mathbb{R}^{N}}^{2} \zeta_{*} d x\right)^{1 / 2}\left(\int_{\Omega} \zeta_{*}^{-1} d x\right)^{1 / 2} \\
\leq C\left(\int_{\Omega}(\nabla y, A(x) \nabla y)_{\mathbb{R}^{N}} d x\right)^{1 / 2} \leq C\|y\|_{A^{\prime}}
\end{gathered}
$$

the space $W_{A}$ is complete with respect to the norm $\|\cdot\|_{A}$. It is clear that $H_{A} \subset W_{A}$, and $W_{A}, H_{A}$ are Hilbert spaces. If the eigenvalues $\left\{\lambda_{1}^{A}, \ldots, \lambda_{N}^{A}\right\}$ of $A: \Omega \rightarrow \mathbb{S}^{N}$ are bounded between two positive constants, then it is easy to verify that $W_{A}=H_{A}$. However, for a "typical" matrix $A \in \mathfrak{M}_{\alpha}^{\beta}(\Omega)$ the space of smooth functions $C_{0}^{\infty}(\Omega)$ is not dense in $W_{A}$. Hence, the identity $W_{A}=H_{A}$ is not always valid (for the corresponding examples in the case when $A(x)=\rho(x) I$, we refer the reader to $[30,31])$.

Weak Compactness Criterion in $L^{1}\left(\Omega ; \mathbb{S}^{N}\right)$. Throughout the paper we will often use the concept of weak and strong convergence in $L^{1}\left(\Omega ; \mathbb{S}^{N}\right)$. Let $\left\{A_{\varepsilon}\right\}_{\varepsilon>0}$ be a bounded sequence of matrices in $L^{1}\left(\Omega ; \mathbb{S}^{N}\right)$. We recall that $\left\{A_{\varepsilon}\right\}_{\mathcal{E}>0}$ is called equi-integrable on $\Omega$, if for any $\delta>0$ there is a $\tau=$ $\tau(\delta)$ such that $\int_{S}\left\|A_{\varepsilon}\right\|_{\mathbb{S}^{N}} d x<\delta$ for every measurable subset $S \subset \Omega$ of Lebesgue measure $|S|<\tau$. Then the following assertions are equivalent for $L^{1}\left(\Omega ; \mathbb{S}^{N}\right)$-bounded sequences:

(i) the sequence $\left\{A_{k}\right\}_{k \in \mathbb{N}}$ is weakly compact in $L^{1}\left(\Omega ; \mathbb{S}^{N}\right)$;

(ii) the sequence $\left\{A_{k}\right\}_{k \in \mathbb{N}}$ is equi-integrable.

Lemma 2 (Lebesgue's Theorem). If a sequence $\left\{A_{k}\right\}_{k \in \mathbb{N}} \subset$ $L^{1}\left(\Omega ; \mathbb{S}^{N}\right)$ is equi-integrable and $A_{k} \rightarrow A$ almost everywhere in $\Omega$, then $A_{k} \rightarrow A$ in $L^{1}\left(\Omega ; \mathbb{S}^{N}\right)$. 
Functions with Bounded Variation. Let $f: \Omega \rightarrow \mathbb{R}$ be a function of $L^{1}(\Omega)$. Define

$$
\begin{aligned}
\int_{\Omega}|D f|=\sup \left\{\int_{\Omega} f \operatorname{div} \varphi d x: \varphi=\left(\varphi_{1}, \ldots, \varphi_{N}\right)\right. \\
\left.\quad \in C_{0}^{1}\left(\Omega ; \mathbb{R}^{N}\right),|\varphi(x)| \leq 1 \text { for } x \in \Omega\right\},
\end{aligned}
$$

where $\operatorname{div} \varphi=\sum_{i=1}^{N}\left(\partial \varphi_{i} / \partial x_{i}\right)$

According to the Radon-Nikodym theorem, if $\int_{\Omega}|D f|<$ $+\infty$, then the distribution $D f$ is a measure and there exist a vector-valued function $\nabla f \in\left[L^{1}(\Omega)\right]^{N}$ and a measure $D_{s} f$, singular with respect to the $N$-dimensional Lebesgue measure $\mathscr{L}^{N}\lfloor\Omega$ restricted to $\Omega$, such that

$$
D f=\nabla f \mathscr{L}^{N}\left\lfloor\Omega+D_{s} f .\right.
$$

Definition 3. A function $f \in L^{1}(\Omega)$ is said to have a bounded variation in $\Omega$ if $\int_{\Omega}|D f|<+\infty$. By $B V(\Omega)$, we denote the space of all functions in $L^{1}(\Omega)$ with bounded variation.

Under the norm $\|f\|_{B V(\Omega)}=\|f\|_{L^{1}(\Omega)}+\int_{\Omega}|D f|, B V(\Omega)$ is a Banach space. The following compactness result for $B V$ functions is well-known.

Proposition 4. Uniformly bounded sets in BV-norm are relatively compact in $L^{1}(\Omega)$.

Definition 5. A sequence $\left\{f_{k}\right\}_{k=1}^{\infty} \subset B V(\Omega)$ weakly converges to some $f \in B V(\Omega)$, and we write $f_{k} \rightarrow f$ if and only if the following two conditions hold: $f_{k} \rightarrow f$ strongly in $L^{1}(\Omega)$, and $D f_{k} \rightarrow D f$ weakly-* in the space of Radon measures $M\left(\Omega ; \mathbb{R}^{N}\right)$; that is,

$$
\lim _{k \rightarrow \infty} \int_{\Omega}\left(\vec{\varphi}, D f_{k}\right)_{\mathbb{R}^{N}}=\int_{\Omega}(\vec{\varphi}, D f)_{\mathbb{R}^{N}} \quad \forall \vec{\varphi} \in C_{0}\left(\mathbb{R}^{N}\right)^{N}
$$

In the following proposition, we give a compactness result related to this convergence, together with lower semicontinuity (see [32]).

Proposition 6. Let $\left\{f_{k}\right\}_{k=1}^{\infty}$ be a sequence in $B V(\Omega)$ strongly converging to some $f$ in $L^{1}(\Omega)$ and satisfying $\sup _{k \in \mathbb{N}} \int_{\Omega}\left|D f_{k}\right|<+\infty$. Then

(i) $f \in B V(\Omega)$ and $\int_{\Omega}|D f| \leq \liminf _{k \rightarrow \infty} \int_{\Omega}\left|D f_{k}\right|$;

(ii) $f_{k} \rightarrow f$ in $B V(\Omega)$.

Elliptic Variational Inequalities. Following Lions [24], let us cite a well-known result concerning solvability and solution uniqueness for variational inequalities which will be useful in the sequel.
Lemma 7 (see [24, Theorem 8.3]). Let $V$ be a Banach space and $K \subset V$ be a closed convex subset. Let $B: K \rightarrow V^{*}$ be a strictly monotone operator and $f \in V^{*}$ be a given element of the dual space. Then the following variational problem: to find an element $y \in K$ such that

$$
\langle B y, v-y\rangle_{V} \geq\langle f, v-y\rangle_{V} \quad \forall v \in K
$$

admits a unique solution provided operator $B$ is corcive in the following sense: there exists an element $v_{0} \in K$ such that

$$
\frac{\left\langle B y, y-v_{0}\right\rangle_{V}}{\|y\|_{V}} \longrightarrow+\infty \quad \text { as }\|y\|_{V} \longrightarrow \infty, y \in K .
$$

\section{3. $\mathbb{S}^{N}$-Valued Radon Measures and Weak Convergence in Variable $L^{2}$-Spaces}

By a nonnegative Radon measure on $\Omega$ we mean a nonnegative Borel measure which is finite on every compact subset of $\Omega$. The space of all nonnegative Radon measures on $\Omega$ will be denoted by $M_{+}(\Omega)$. According to the Riesz theory, each Radon measure $\mu \in M_{+}(\Omega)$ can be interpreted as an element of the dual space to space $C_{0}(\Omega)$ of all continuous functions with compact support. Let $M\left(\Omega ; \mathbb{S}^{N}\right)$ denote the space of all $\mathbb{S}^{N}$-valued nonnegative Radon measures. Then $\vec{\mu}=$ $\left[\mu_{i j}\right] \in M\left(\Omega ; \mathbb{S}^{N}\right) \Leftrightarrow \mu_{i j} \in\left(C_{0}(\Omega)\right)^{*}, i, j=1, \ldots, N$.

Let $\vec{\mu}$ and $\left\{\vec{\mu}_{k}\right\}_{k \in \mathbb{N}}$ be matrix-valued nonnegative Radon measures. We say that $\left\{\vec{\mu}_{k}\right\}_{k \in \mathbb{N}}$ weakly-* converges to $\vec{\mu}$ in $M\left(\Omega ; \mathbb{S}^{N}\right)$ if

$$
\lim _{k \rightarrow \infty} \int_{\Omega} \vec{\varphi} \cdot d \vec{\mu}_{k}=\int_{\Omega} \vec{\varphi} \cdot d \vec{\mu} \quad \forall \vec{\varphi} \in C_{0}\left(\Omega ; \mathbb{S}^{N}\right) .
$$

A typical example of such measures is

$$
d \vec{\mu}_{k}=A_{k}(x) d x, \quad d \vec{\mu}=A(x) d x,
$$

where

$$
\begin{gathered}
A_{k}, A \in \mathfrak{M}_{\alpha}^{\beta}(\Omega) \cap L^{1}\left(\Omega ; \mathbb{S}^{N}\right), \\
A_{k} \rightarrow A \quad \text { in } L^{1}\left(\Omega ; \mathbb{S}^{N}\right),
\end{gathered}
$$

or

$$
\begin{gathered}
A_{k}, A \in \mathfrak{M}_{\alpha}^{\beta}(\Omega) \cap L^{\infty}\left(\Omega ; \mathbb{S}^{N}\right), \\
A_{k} \stackrel{*}{\rightarrow} A \text { in } L^{\infty}\left(\Omega ; \mathbb{S}^{N}\right) .
\end{gathered}
$$

As we will see later (see Lemma 15), the sets $\mathfrak{M}_{\alpha}^{\beta}(\Omega) \cap$ $L^{1}\left(\Omega ; \mathbb{S}^{N}\right)$ are sequentially closed with respect to strong convergence in $L^{1}\left(\Omega ; \mathbb{S}^{N}\right)$.

In this section, we suppose that the measures $\vec{\mu}$ and $\left\{\vec{\mu}_{k}\right\}_{k \in \mathbb{N}}$ are defined by (21) and $\vec{\mu}_{k} \stackrel{*}{\rightarrow} \vec{\mu}$ in $M\left(\Omega ; \mathbb{S}^{N}\right)$. Further, we will use $L^{2}(\Omega, A d x)^{N}$ to denote the set of measurable vector-valued functions $\mathbf{f} \in \mathbb{R}^{N}$ on $\Omega$ such that

$$
\|\mathbf{f}\|_{L^{2}(\Omega, A d x)^{N}}=\left(\int_{\Omega}(\mathbf{f}, A(x) \mathbf{f})_{\mathbb{R}^{N}} d x\right)^{1 / 2}<+\infty .
$$


As follows from estimate (14), any vector-valued function of $L^{2}(\Omega, A d x)^{N}$ is Lebesgue integrable on $\Omega$.

We say that a sequence $\left\{\mathbf{v}_{k} \in L^{2}\left(\Omega, A_{k} d x\right)^{N}\right\}_{k \in \mathbb{N}}$ is bounded if

$$
\limsup _{k \rightarrow \infty} \int_{\Omega}\left(\mathbf{v}_{k}, A_{k}(x) \mathbf{v}_{k}\right)_{\mathbb{R}^{N}} d x<+\infty
$$

Definition 8. Let $A$ and $\left\{A_{k}\right\}_{k \in \mathbb{N}}$ be matrices satisfying conditions (22). A bounded sequence $\left\{\mathbf{v}_{k} \in L^{2}\left(\Omega, A_{k} d x\right)^{N}\right\}_{k \in \mathbb{N}}$ is weakly convergent to a function $\mathbf{v} \in L^{2}(\Omega, A d x)^{N}$ in the variable space $L^{2}\left(\Omega, A_{k} d x\right)^{N}$ if

$$
\begin{array}{r}
\lim _{k \rightarrow \infty} \int_{\Omega}\left(\vec{\varphi}, A_{k}(x) \mathbf{v}_{k}\right)_{\mathbb{R}^{N}} d x=\int_{\Omega}(\vec{\varphi}, A(x) \mathbf{v})_{\mathbb{R}^{N}} d x \\
\forall \vec{\varphi} \in C_{0}^{\infty}(\Omega)^{N} .
\end{array}
$$

The main property concerning the weak convergence in $L^{2}\left(\Omega, A_{k} d x\right)^{N}$ can be expressed as follows (see for comparison [33]).

Proposition 9 (see [29]). Let $A$ and $\left\{A_{k}\right\}_{k \in \mathbb{N}}$ be matrices satisfying conditions (22). If a sequence $\left\{\mathbf{v}_{k} \in L^{2}\left(\Omega, A_{k} d x\right)^{N}\right\}_{k \in \mathbb{N}}$ is bounded, then it is compact in the sense of weak convergence in $L^{2}\left(\Omega, A_{k} d x\right)^{N}$.

The next property of weak convergence in $L^{2}(\Omega$, $\left.A_{k} d x\right)^{N}$ is the lower semicontinuity of the variable $L^{2}$ norm.

Proposition 10 (see [29]). If the sequence $\left\{\mathbf{v}_{k} \in L^{2}(\Omega\right.$, $\left.\left.A_{k} d x\right)^{N}\right\}_{k \in \mathbb{N}}$ converges weakly to $\mathbf{v} \in L^{2}(\Omega, A d x)^{N}$, then

$$
\liminf _{k \rightarrow \infty} \int_{\Omega}\left(\mathbf{v}_{k}, A_{k}(x) \mathbf{v}_{k}\right)_{\mathbb{R}^{N}} d x \geq \int_{\Omega}(\mathbf{v}, A(x) \mathbf{v})_{\mathbb{R}^{N}} d x .
$$

Definition 11. A sequence $\left\{\mathbf{v}_{k} \in L^{2}\left(\Omega, A_{k} d x\right)^{N}\right\}_{k \in \mathbb{N}}$ is said to be strongly convergent to a function $\mathbf{v} \in L^{2}(\Omega, A d x)^{N}$ if

$$
\lim _{k \rightarrow \infty} \int_{\Omega}\left(\mathbf{b}_{k}, A_{k}(x) \mathbf{v}_{k}\right)_{\mathbb{R}^{N}} d x=\int_{\Omega}(\mathbf{b}, A(x) \mathbf{v})_{\mathbb{R}^{N}} d x
$$

whenever $\mathbf{b}_{k} \rightarrow \mathbf{b}$ in $L^{2}\left(\Omega, A_{k} d x\right)^{N}$ as $k \rightarrow \infty$. We have the following property of strong convergence in the variable $L^{2}\left(\Omega, A_{k} d x\right)^{N}$-spaces.

Proposition 12 (see [29]). Weak convergence of a sequence $\left\{\mathbf{v}_{k} \in L^{2}\left(\Omega, A_{k} d x\right)^{N}\right\}_{k \in \mathbb{N}}$ to $\mathbf{v} \in L^{2}(\Omega, A d x)^{N}$ and

$$
\lim _{k \rightarrow \infty} \int_{\Omega}\left(\mathbf{v}_{k}, A_{k}(x) \mathbf{v}_{k}\right)_{\mathbb{R}^{N}} d x=\int_{\Omega}(\mathbf{v}, A(x) \mathbf{v})_{\mathbb{R}^{N}} d x
$$

is equivalent to strong convergence of $\left\{\mathbf{v}_{k}\right\}_{k \in \mathbb{N}}$ in $L^{2}\left(\Omega, A_{k} d x\right)^{N}$ to $\mathbf{v} \in L^{2}(\Omega, A d x)^{N}$.

\section{Auxiliary Results}

Following in many aspects Kogut and Leugering [29], we provide some properties of the set $\Psi_{*} \subset L^{1}(\Omega)$ defined in (7), and give some auxiliary results.
Lemma 13 (see [29]). Let $\left\{\zeta_{*, n}\right\}_{n \in \mathbb{N}}$ be any sequence in $\Psi_{*}$. Then, there is an element $\zeta_{*} \in L^{1}(\Omega)$ such that, within a subsequence of $\left\{\zeta_{*, n}\right\}_{n \in \mathbb{N}}$, we have

$$
\begin{gathered}
\zeta_{*, n} \longrightarrow \zeta_{*} \quad \text { in } L^{1}(\Omega), \quad \zeta_{*} \in \Psi_{*}, \\
\zeta_{*, n}^{-1} \longrightarrow \zeta_{*}^{-1} \text { in } L^{1}(\Omega),
\end{gathered}
$$

$\zeta_{*, n}^{-1} \longrightarrow \zeta_{*}^{-1} \quad$ in variable space $L^{2}\left(\Omega, \zeta_{*, n} d x\right)$.

For our further analysis, we make use of the following concept.

Definition 14. We say that a bounded sequence

$$
\left\{\left(A_{n}, y_{n}\right) \in L^{1}\left(\Omega ; \mathbb{S}^{N}\right) \times H\left(\Omega ; A_{n} d x\right)\right\}_{n \in \mathbb{N}}
$$

$w$-converges to $(A, y) \in L^{1}\left(\Omega ; \mathbb{S}^{N}\right) \times W_{0}^{1,1}(\Omega)$ as $n \rightarrow \infty$ if

$$
\begin{gathered}
A_{n} \longrightarrow A \quad \text { in } L^{1}\left(\Omega ; \mathbb{S}^{N}\right), \\
y_{n} \rightarrow y \quad \text { in } L^{2}(\Omega),
\end{gathered}
$$

$\nabla y_{n} \rightarrow \nabla y \quad$ in the variable space $L^{2}\left(\Omega, A_{n} d x\right)^{N}$;

therefore,

$$
\begin{gathered}
\lim _{n \rightarrow \infty} \int_{\Omega} A_{n} \cdot \vec{\eta} d x=\int_{\Omega} A \cdot \vec{\eta} d x \quad \forall \vec{\eta} \in L^{\infty}\left(\Omega ; \mathbb{S}^{N}\right), \\
\lim _{n \rightarrow \infty} \int_{\Omega} y_{n} \lambda d x=\int_{\Omega} y \lambda d x \quad \forall \lambda \in L^{2}(\Omega), \\
\lim _{n \rightarrow \infty} \int_{\Omega}\left(\vec{\xi}, A_{n} \nabla y_{n}\right)_{\mathbb{R}^{N}} d x=\int_{\Omega}(\vec{\xi}, A \nabla y)_{\mathbb{R}^{N}} d x \\
\forall \vec{\xi} \in C_{0}^{\infty}(\Omega)^{N} .
\end{gathered}
$$
result.

In order to motivate this definition, we give the following

Lemma 15. Let $\left\{\left(A_{n}, y_{n}\right) \in L^{1}\left(\Omega ; \mathbb{S}^{N}\right) \times H\left(\Omega ; A_{n} d x\right)\right\}_{n \in \mathbb{N}}$ be a sequence such that

(i) the sequence $\left\{y_{n} \in H\left(\Omega ; A_{n} d x\right)\right\}_{n \in \mathbb{N}}$ is bounded; that is,

$$
\sup _{n \in \mathbb{N}} \int_{\Omega}\left(y_{n}^{2}+\left(\nabla y_{n}, A_{n} \nabla y_{n}\right)\right) d x<+\infty ;
$$

(ii) $\left\{A_{n}\right\}_{n \in \mathbb{N}} \subset \mathfrak{M}_{\alpha}^{\beta}(\Omega)$ and there exists a matrix-valued function $A(x) \in \mathbb{S}^{N}$ such that

$$
\begin{array}{r}
A_{n} \longrightarrow A, \quad A_{n}^{-1} \longrightarrow A^{-1} \quad \text { in } L^{1}\left(\Omega ; \mathbb{S}^{N}\right) \\
\text { as } n \longrightarrow \infty .
\end{array}
$$

Then, $A \in \mathfrak{M}_{\alpha}^{\beta}(\Omega) \cap L^{1}\left(\Omega ; \mathbb{S}^{N}\right)$; and the original sequence is relatively compact with respect to $w$-convergence. Moreover, each $w$-limit pair $(A, y)$ belongs to the space $L^{1}\left(\Omega ; \mathbb{S}^{N}\right) \times$ $H(\Omega ; A d x)$. 
Proof. We note that (36)-(37), (4), and (13)-(14) immediately imply the boundedness of the original sequence in $L^{1}\left(\Omega ; \mathbb{S}^{N}\right) \times W^{1,1}(\Omega)$. Moreover, due to (37), we have

$$
d \vec{\mu}_{n}:=A_{n} d x \stackrel{*}{\rightarrow} A d x=: d \vec{\mu} \quad \text { in } \mathscr{M}\left(\Omega ; \mathbb{S}^{N}\right) .
$$

Thus, the compactness criterium for weak convergence in variable spaces (see Proposition 9) and (36) imply the existence of a pair $(y, \mathbf{v}) \in L^{2}(\Omega) \times L^{2}(\Omega, A d x)^{N}$ such that, within a subsequence of $\left\{y_{n}\right\}_{n \in \mathbb{N}}$,

$$
\begin{gathered}
y_{n} \rightarrow y \quad \text { in } L^{2}(\Omega), \\
\nabla y_{n} \rightarrow \mathbf{v} \quad \text { in variable space } L^{2}\left(\Omega, A_{n} d x\right)^{N} .
\end{gathered}
$$

Our aim is to show that $A \in \mathfrak{M}_{\alpha}^{\beta}(\Omega), \mathbf{v}=\nabla y$, and $y \in$ $H(\Omega ; A d x)$. It is clear that $A(x) \in \mathbb{S}^{N}$ and this matrix satisfies (8). Since $A_{n} \in \mathfrak{M}_{\alpha}^{\beta}(\Omega) \cap L^{1}\left(\Omega ; \mathbb{S}^{N}\right)$ for all $n \in \mathbb{N}$, it follows that there is a sequence $\left\{\zeta_{*, n}\right\}_{n \in \mathbb{N}}$ in $\Psi_{*}$ such that

$$
\zeta_{*, n}(x) I \leq A_{n}(x) I \leq \beta(x) I
$$$$
\text { a.e. in } \Omega \quad \forall k \in\{1, \ldots, N\} \text {. }
$$

Then, by $L^{1}$-compactness of the set $\Psi_{*}$, there exists an element $\zeta_{*} \in \Psi_{*}$ such that $\zeta_{*, n} \rightarrow \zeta_{*}$ in $L^{1}(\Omega)$ as $n \rightarrow \infty$. Moreover, Lemma 13 implies strong convergence

$$
\zeta_{*, n}^{-1} \longrightarrow \zeta_{*}^{-1} \quad \text { in } L^{1}(\Omega)
$$

and (7). Hence, passing to the limit in (41) as $n \rightarrow \infty$, we come to (9). Thus, $A \in \mathfrak{M}_{\alpha}^{\beta}(\Omega)$ and the limit matrix $A(x) \in$ $\mathbb{S}^{N}$ satisfies (10)-(11).

For our further analysis, we fix any test function $\vec{\varphi} \in$ $C_{0}^{\infty}(\Omega)^{N}$ and make use of the following equality:

$$
\begin{aligned}
\int_{\Omega}\left(A_{n}^{-1} \vec{\varphi}, A_{n} \vec{\psi}\right)_{\mathbb{R}^{N}} d x & =\int_{\Omega}(\vec{\varphi}, \vec{\psi})_{\mathbb{R}^{N}} d x \\
& =\int_{\Omega}\left(A^{-1} \vec{\varphi}, A \vec{\psi}\right)_{\mathbb{R}^{N}} d x,
\end{aligned}
$$

which is obviously true for each $\vec{\psi} \in C_{0}^{\infty}(\Omega)^{N}$ and for all $n \in$ $\mathbb{N}$. Since

$$
\begin{gathered}
\limsup _{n \rightarrow \infty} \int_{\Omega}\left(A_{n}^{-1} \vec{\varphi}, A_{n} A_{n}^{-1} \vec{\varphi}\right)_{\mathbb{R}^{N}} d x \\
\quad=\limsup _{n \rightarrow \infty} \int_{\Omega}\left(\vec{\varphi}, A_{n}^{-1} \vec{\varphi}\right)_{\mathbb{R}^{N}} d x \\
\leq \limsup _{n \rightarrow \infty} \int_{\Omega} \zeta_{*, n}^{-1}\|\vec{\varphi}\|_{\mathbb{R}^{N}}^{2} d x \\
\quad \text { by (42) } \\
\stackrel{=}{=} \int_{\Omega} \zeta_{*}^{-1}\|\vec{\varphi}\|_{\mathbb{R}^{N}}^{2} d x \\
\leq\|\vec{\varphi}\|_{C(\Omega)^{N}}^{2}\left\|\zeta_{*}^{-1}\right\|_{L^{1}(\Omega)}<+\infty,
\end{gathered}
$$

it follows that the sequence $\left\{A_{n}^{-1} \vec{\varphi} \in L^{2}\left(\Omega, A_{n} d x\right)^{N}\right\}_{n \in \mathbb{N}}$ is bounded. Consequently, combining this fact with (43), we conclude that $A_{n}^{-1} \vec{\varphi} \rightarrow A^{-1} \vec{\varphi}$ in the variable space $L^{2}\left(\Omega, A_{n} d x\right)^{N}$ (see Definition 8). At the same time, strong convergence in (37) implies the relation

$$
\begin{aligned}
& \lim _{n \rightarrow \infty} \int_{\Omega}\left(A_{n}^{-1} \vec{\varphi}, A_{n} A_{n}^{-1} \vec{\varphi}\right)_{\mathbb{R}^{N}} d x \\
& \quad=\lim _{n \rightarrow \infty} \int_{\Omega}\left(\vec{\varphi}, A_{n}^{-1} \vec{\varphi}\right)_{\mathbb{R}^{N}} d x \\
& \quad=\int_{\Omega}\left(\vec{\varphi}, A^{-1} \vec{\varphi}\right)_{\mathbb{R}^{N}} d x=\int_{\Omega}\left(A^{-1} \vec{\varphi}, A A^{-1} \vec{\varphi}\right)_{\mathbb{R}^{N}} d x .
\end{aligned}
$$

Hence (see Proposition 12),

$$
\begin{array}{r}
A_{n}^{-1} \vec{\varphi} \longrightarrow A^{-1} \vec{\varphi} \quad \text { strongly in } L^{2}\left(\Omega, A_{n} d x\right)^{N} \\
\forall \vec{\varphi} \in C_{0}^{\infty}(\Omega)^{N} .
\end{array}
$$

Further, we note that for every measurable subset $S \subset \Omega$, the estimate

$$
\begin{aligned}
\int_{S} \| & \nabla y_{n} \|_{\mathbb{R}^{N}} d x \\
& \leq\left(\int_{S}\left\|\nabla y_{n}\right\|_{\mathbb{R}^{N}}^{2} \zeta_{*, n} d x\right)^{1 / 2}\left(\int_{S} \zeta_{*, n}^{-1} d x\right)^{1 / 2} \\
& \leq\left(\int_{\Omega}\left(\nabla y_{n}, A_{n}(x) \nabla y_{n}\right)_{\mathbb{R}^{N}} d x\right)^{1 / 2}\left(\int_{S} \zeta_{*, n}^{-1} d x\right)^{1 / 2} \\
& \leq C\left(\int_{S} \zeta_{*, n}^{-1} d x\right)^{1 / 2}
\end{aligned}
$$

implies equi-integrability of the family $\left\{\left\|\nabla y_{n}\right\|_{\mathbb{R}^{N}}\right\}_{n \in \mathbb{N}^{*}}$. Hence, $\quad\left\{\left\|\nabla y_{n}\right\|_{\mathbb{R}^{N}}\right\}_{n \in \mathbb{N}}$ is weakly compact in $L^{1}(\Omega)$, which means the weak compactness of the vector-valued sequence $\left\{\nabla y_{n}\right\}_{n \in \mathbb{N}}$ in $L^{1}(\Omega)^{N}$. As a result, by the properties of the strong convergence in variable spaces, we obtain

$$
\begin{array}{r}
\int_{\Omega}\left(\vec{\xi}, \nabla y_{n}\right)_{\mathbb{R}^{N}} d x=\int_{\Omega}\left(A_{n}^{-1} \vec{\xi}, A_{n} \nabla y_{n}\right)_{\mathbb{R}^{N}} d x \\
\text { by (28), (40), and }(46) \\
\int_{\Omega}\left(A^{-1} \vec{\xi}, A \mathbf{v}\right)_{\mathbb{R}^{N}} d x \\
=\int_{\Omega}(\vec{\xi}, \mathbf{v})_{\mathbb{R}^{N}} d x \\
\forall \vec{\xi} \in C_{0}^{\infty}(\Omega)^{N} .
\end{array}
$$

Thus, in view of the weak compactness property of $\left\{\nabla y_{n}\right\}_{n \in \mathbb{N}}$ in $L^{1}(\Omega)^{N}$, we conclude that

$$
\nabla y_{n} \rightarrow \mathbf{v} \quad \text { in } L^{1}\left(\Omega ; \mathbb{R}^{N}\right) \quad \text { as } n \longrightarrow \infty .
$$

Since $y_{n} \in W_{0}^{1,1}(\Omega)$ for all $n \in \mathbb{N}$ and the Sobolev space $W_{0}^{1,1}(\Omega)$ is complete, (39) and (49) imply $\nabla y=\mathbf{v}$, and consequently $y \in W_{0}^{1,1}(\Omega)$. It should be also observed that 
(39)-(40) guarantee the finiteness of the norm $\|y\|_{A}$ (see (4)), hence $y \in W(\Omega ; A d x)$ (to elaborate Propositions 9, 10, 12 and Lemma 13, for the sake of reader's convenience, we closely followed the idea of [29]). However, to show that $y \in H(\Omega ; A d x)$, we must be sure that there exists a sequence of smooth functions from $C_{0}^{\infty}(\Omega)$ strongly converging in $W(\Omega ; A d x)$ to this element.

Since for all $n \in \mathbb{N}, y_{n} \in H\left(\Omega ; A_{n} d x\right)$, there exist sequences $\left\{\varphi_{k}^{n}\right\}_{k \in \mathbb{N}} \subset C_{0}^{\infty}(\Omega)$, such that $\varphi_{k}^{n} \rightarrow y_{n}$ strongly in $H\left(\Omega ; A_{n} d x\right)$ as $k \rightarrow \infty$. Let us show that the sequence $\left\{\varphi_{n}^{n}\right\}_{n \in \mathbb{N}}$, chosen with respect to diagonalization procedure, converges weakly to $y$ in $W(\Omega ; A d x)$. To this end, it is enough to prove that $\varphi_{n}^{n} \rightarrow y$ in $L^{2}(\Omega)$ and $\nabla \varphi_{n}^{n} \rightarrow \nabla y$ in $L^{2}(\Omega ; A d x)^{N}$.

Indeed, for an arbitrary $\psi \in C_{0}^{\infty}(\Omega)$, we have

$$
\begin{aligned}
\left|\int_{\Omega}\left(y-\varphi_{n}^{n}\right) \psi d x\right| \leq & \left|\int_{\Omega}\left(y-y_{n}\right) \psi d x\right| \\
& +\left|\int_{\Omega}\left(y_{n}-\varphi_{n}^{n}\right) \psi d x\right| \\
\text { as } n \longrightarrow 0 & \longrightarrow \infty
\end{aligned}
$$

since $y_{n} \rightarrow y$ in $L^{2}(\Omega)$, as $n \rightarrow \infty$ and for any $\varepsilon>0$ there exists $N_{0} \in \mathbb{N}$ such that $\left|\int_{\Omega}\left(y_{n}-\varphi_{n}^{n}\right) \psi d x\right|<\varepsilon$ for all $n>N_{0}$. Similarly, for an arbitrary $\vec{\psi} \in C_{0}^{\infty}(\Omega)^{N}$, we have

$$
\begin{aligned}
& \mid \int_{\Omega}(\left.\left(\nabla y-\nabla \varphi_{n}^{n}\right), \vec{\psi}\right)_{\mathbb{R}^{N}} d x \mid \\
& \leq\left|\int_{\Omega}(A \nabla y, \vec{\psi})_{\mathbb{R}^{N}} d x-\int_{\Omega}\left(A_{n} \nabla y_{n}, \vec{\psi}\right)_{\mathbb{R}^{N}} d x\right| \\
&+\left|\int_{\Omega}\left(A_{n}\left(\nabla y_{n}-\nabla \varphi_{n}^{n}\right), \vec{\psi}\right)_{\mathbb{R}^{N}} d x\right| \\
&+\left|\int_{\Omega}\left(\left(A_{n}-A\right) \nabla \varphi_{n}^{n}, \vec{\psi}\right)_{\mathbb{R}^{N}} d x\right| \longrightarrow 0, \\
& \text { as } n \longrightarrow \infty .
\end{aligned}
$$

It is only the convergence to zero of the third summand that should be explained here:

$$
\begin{aligned}
\mid \int_{\Omega}( & \left.\left(A_{n}-A\right) \nabla \varphi_{n}^{n}, \vec{\psi}\right)_{\mathbb{R}^{N}} d x \mid \\
\leq & \left(\int_{\Omega}\left(\left|A_{n}-A\right| \nabla \varphi_{n}^{n}, \nabla \varphi_{n}^{n}\right)_{\mathbb{R}^{N}} d x\right)^{1 / 2} \\
& \times\left(\int_{\Omega}\left(\left|A_{n}-A\right| \vec{\psi}, \vec{\psi}\right)_{\mathbb{R}^{N}} d x\right)^{1 / 2}=J_{1} \cdot J_{2} .
\end{aligned}
$$

Obviously, $J_{2} \rightarrow 0$ as $n \rightarrow \infty$. It is left to prove, that $J_{1} \leq$ C. Indeed,

$$
\begin{aligned}
& \int_{\Omega}\left(\left|A_{n}-A\right| \nabla \varphi_{n}^{n}, \nabla \varphi_{n}^{n}\right)_{\mathbb{R}^{N}} d x \\
& \leq \int_{\Omega}\left(A_{n} \nabla \varphi_{n}^{n}, \nabla \varphi_{n}^{n}\right)_{\mathbb{R}^{N}} d x+\int_{\Omega}\left(A \nabla \varphi_{n}^{n}, \nabla \varphi_{n}^{n}\right)_{\mathbb{R}^{N}} d x \\
& \leq \int_{\Omega}\left(A_{n} \nabla \varphi_{n}^{n}, \nabla \varphi_{n}^{n}\right)_{\mathbb{R}^{N}} d x \\
&+\int_{\Omega} \beta \zeta_{a d}^{-1}\left(A_{n} \nabla \varphi_{n}^{n}, \nabla \varphi_{n}^{n}\right)_{\mathbb{R}^{N}} d x \\
& \leq\left(1+\left\|\beta \zeta_{a d}^{-1}\right\|_{L^{\infty}(\Omega)}\right)\left(\int_{\Omega}\left(A_{n} \nabla \varphi_{n}^{n}, \nabla \varphi_{n}^{n}\right)_{\mathbb{R}^{N}} d x\right. \\
& \pm \int_{\Omega}\left(A_{n} \nabla y_{n}, \nabla y_{n}\right)_{\mathbb{R}^{N}} d x
\end{aligned}
$$

$\leq C$

since $\left\|\nabla \varphi_{n}^{n}\right\|_{L^{2}\left(\Omega ; A_{n} d x\right)}^{2}-\left\|\nabla y_{n}\right\|_{L^{2}\left(\Omega ; A_{n} d x\right)}^{2} \rightarrow 0$ as $n \rightarrow \infty$ and $\left\{\left\|\nabla y_{n}\right\|_{L^{2}\left(\Omega ; A_{n} d x\right)}^{2}\right\}_{n \in \mathbb{N}}$ is a bounded sequence in $\mathbb{R}$.

Hence, we proved the existence of the sequence from $C_{0}^{\infty}(\Omega)$ which is weakly convergent in $W(\Omega ; A d x)$, to the element $y$. However, it is well known that due to Mazur's lemma in $W(\Omega ; A d x)$ there exists a sequence, strongly convergent to $y$, which is constructed as a certain linear combinations of elements from $\left\{\varphi_{n}^{n}\right\}_{n \in \mathbb{N}}$. Linear combinations of smooth functions are smooth as well. Hence, as the strong limit of the sequence from $C_{0}^{\infty}(\Omega)$, the element $y$ belongs to the space $H(\Omega ; A d x)$.

\section{Setting of the Optimal Control Problem}

Let $M \in \mathbb{S}^{N}$ be a given constant matrix satisfying the condition

$$
(M \xi, \xi)_{\mathbb{R}^{N}} \geq m\|\xi\|_{\mathbb{R}^{N}}^{2} \quad \text { for some } m>0, \forall \xi \in \mathbb{R}^{N} .
$$

Let $K$ be a convex closed subset of the space $W_{0}^{1,1}(\Omega)$ such that $K \cap C_{0}^{\infty}(\Omega) \neq \emptyset$, and let $f \in L^{2}(\Omega)$ be a given distribution. Having fixed $A(x) \in L^{1}\left(\Omega ; \mathbb{S}^{N}\right)$, we denote by $K_{A}$ the closure of $\{K \cap H(\Omega ; A d x)\}$ with respect to the norm $\|\cdot\|_{A}$, and consider the following elliptic variational inequality: to find an element $y \in K_{A}$ such that

$$
\begin{array}{r}
\langle-\operatorname{div}(A(x) \nabla y)+y, v-y\rangle_{H_{A}} \geq\langle f, v-y\rangle_{H_{A}}, \\
\forall v \in K_{A} .
\end{array}
$$

Remark 16. The distinctive feature of this variational problem is the following fact: choosing a matrix of coefficients $A(x) \epsilon$ $L^{1}\left(\Omega ; \mathbb{S}^{N}\right)$, we choose a space in which the problem is considered; or, speaking in terms of optimization problem in coefficients for given inequality, changing a control matrix, we change not only the state or even the space of states but also the control object itself. 
To introduce the class of admissible controls in coefficients, we adopt the following concept.

Definition 17. We say that a matrix-valued function $A=$ $A(x) \in L^{1}\left(\Omega ; \mathbb{S}^{N}\right)$ is an admissible control for variational inequality (55) (it is written as $A \in \mathfrak{\mathfrak { A }}_{a d}$ ) if

$$
\begin{gathered}
A \in B V\left(\Omega ; \mathbb{S}^{N}\right), \quad A \in \mathfrak{M}_{\alpha}^{\beta}(\Omega), \\
\int_{\Omega} A(x) d x=M .
\end{gathered}
$$

Hereinafter, we assume that the set $\mathfrak{A}_{a d}$ is nonempty.

The optimal control problem we consider here is to minimize the discrepancy (tracking error) between a given distribution $y_{d} \in L^{2}(\Omega)$ and the solution of elliptic variational inequality (55)-(56) by choosing an appropriate coefficients matrix $A \in \mathfrak{A}_{a d}$. More precisely, we are concerned with the following optimal control problem:

$$
\text { Minimize } \begin{aligned}
\{I(A, y)= & \int_{\Omega}\left|y(x)-y_{d}(x)\right|^{2} d x \\
& +\int_{\Omega}(\nabla y(x), A(x) \nabla y(x))_{\mathbb{R}^{N}} d x \\
& \left.+\sum_{i, j=1}^{N} \int_{\Omega}\left|D a_{i j}(x)\right|\right\}
\end{aligned}
$$

subject to the constraints (55)-(56).

Remark 18. The second term in (57) plays a special role in this problem. Its appearance in the cost function (57) is motivated by the fact that there are no appropriate a priori estimates in the $H_{A}$-norm for solutions $y=y(A, f)$ of problem (55)-(56). Hence, the term $\int_{\Omega}(\nabla y(x), A(x) \nabla y(x))_{\mathbb{R}^{N}} d x$ together with the first one in (57) ensures the coercivity of the cost function.

We can indicate the set of admissible solutions to the original optimal control problem as follows:

$\Xi=\left\{(A, y) \mid A \in \mathfrak{\mathfrak { A }}_{a d}, y \in H_{A},(A, y)\right.$ are related by $\left.(55)\right\}$

Remark 19. Note that due to (13)-(14), we have the following obvious inclusion for the set of admissible solutions: $\Xi \subset$ $L^{1}\left(\Omega ; \mathbb{S}^{N}\right) \times W_{0}^{1,1}(\Omega)$.

To discuss solvability of variational inequality (55) and uniqueness of its solution, we make use of the following results.
Lemma 20. For every fixed admissible control $A \in \mathfrak{A}_{a d}$, the operator $B_{A}: K_{A} \rightarrow H(\Omega ; A d x)$, defined as

$$
\begin{aligned}
\left\langle B_{A}(y), v\right\rangle_{H_{A}}= & \sum_{i, j=1}^{N} \int_{\Omega}\left(a_{i j}(x) \frac{\partial y}{\partial x_{j}}\right) \frac{\partial v}{\partial x_{i}} d x \\
& +\int_{\Omega} y v d x, \quad \forall v \in H(\Omega ; A d x),
\end{aligned}
$$

is strictly monotone and coercive.

Proof. Let $A \in \mathfrak{A}_{a d}$ be a fixed matrix. Then the relation

$$
\left\langle B_{A}(y), y\right\rangle_{H_{A}}=\int_{\Omega}(\nabla y, A \nabla y)_{\mathbb{R}^{N}} d x+\int_{\Omega} y^{2} d x=\|y\|_{A}^{2}
$$

implies the strict monotonicity and coercivity property of the operator $B_{A}$. Indeed, by setting in (60) $y=u-v, u, v \in H_{A}$, we immediately obtain

$$
\left\langle B_{A}(u)-B_{A}(v), u-v\right\rangle_{H_{A}}=\|u-v\|_{A}^{2}>0 \quad \forall u \neq v .
$$

Proposition 21. For every control $A \in \mathfrak{\mathfrak { A }}_{a d}$ and every $f \in$ $L^{2}(\Omega)$, there exists a unique solution to degenerate elliptic variational inequality (55)-(56).

Proof. Taking into account Lemmas 7 and 20, it is enough to show that there exists an element $v_{0} \in K_{A}$ such that

$$
\begin{aligned}
& \frac{\left\langle-\operatorname{div}(A(x) \nabla y)+y, y-v_{0}\right\rangle_{H_{A}}}{\|y\|_{A}} \longrightarrow+\infty \\
& \text { as }\|y\|_{A} \longrightarrow \infty, \quad y \in K_{A} .
\end{aligned}
$$

In order to do this, we fix an arbitrary element $v_{0} \in K_{A}$ and a matrix $A \in \mathfrak{A}_{a d}$. Then for all $y \in K_{A}$, in view of estimate (60), we have

$$
\begin{aligned}
&\langle-\operatorname{div}\left.(A(x) \nabla y)+y, y-v_{0}\right\rangle_{H_{A}} \\
&=\|y\|_{A}^{2}-\left\langle-\operatorname{div}(A(x) \nabla y)+y, v_{0}\right\rangle_{H_{A}} \\
& \geq\|y\|_{A}^{2}-\left|\left\langle-\operatorname{div}(A(x) \nabla y)+y, v_{0}\right\rangle_{H_{A}}\right|,
\end{aligned}
$$

where

$$
\begin{aligned}
& \left|\left\langle-\operatorname{div}(A(x) \nabla y)+y, v_{0}\right\rangle_{H_{A}}\right| \\
& \quad=\left|\int_{\Omega}\left(A(x) \nabla y, \nabla v_{0}\right)_{\mathbb{R}^{N}} d x+\int_{\Omega} y v_{0} d x\right|
\end{aligned}
$$




$$
\begin{aligned}
\leq & \left(\int_{\Omega}\left|A^{1 / 2} \nabla y\right|_{\mathbb{R}^{N}}^{2} d x\right)^{1 / 2}\left(\int_{\Omega}\left|A^{1 / 2} \nabla v_{0}\right|_{\mathbb{R}^{N}}^{2} d x\right)^{1 / 2} \\
& +\|y\|_{L^{2}(\Omega)}\left\|v_{0}\right\|_{L^{2}(\Omega)} \\
= & \left(\int_{\Omega}(\nabla y, A \nabla y)_{\mathbb{R}^{N}} d x\right)^{1 / 2}\left(\int_{\Omega}\left(\nabla v_{0}, A \nabla v_{0}\right)_{\mathbb{R}^{N}} d x\right)^{1 / 2} \\
& +\|y\|_{L^{2}(\Omega)}\left\|v_{0}\right\|_{L^{2}(\Omega)} \\
= & \|\nabla y\|_{L^{2}(\Omega ; A d x)^{N}}\left\|v_{0}\right\|_{L^{2}(\Omega ; A d x)^{N}}+\|y\|_{L^{2}(\Omega)}\left\|v_{0}\right\|_{L^{2}(\Omega)} \\
\leq & \|y\|_{A}\left\|v_{0}\right\|_{A^{\prime}} .
\end{aligned}
$$

Combining (63) and (64) we come to the inequality

$$
\begin{aligned}
& \frac{\left\langle-\operatorname{div}(A(x) \nabla y)+y, y-v_{0}\right\rangle_{H_{A}}}{\|y\|_{A}} \geq\|y\|_{A}-\left\|v_{0}\right\|_{A} \longrightarrow+\infty, \\
& \text { as }\|y\|_{A} \longrightarrow \infty \text {. }
\end{aligned}
$$

Hence, problem (55)-(56) satisfies all conditions of Lemma 7. Therefore, for every control $A(x) \in \mathfrak{A}_{a d}$ and every $f \in L^{2}(\Omega)$, the considered problem has a unique solution.

Thus, as follows from Proposition 21, the set of admissible solutions $\Xi$ is nonempty. Hence, the minimization problem $\inf _{(A, y) \in \Xi} I(A, y)$ is regular. We say that a pair $\left(A^{0}, y^{0}\right) \in$ $L^{1}\left(\Omega ; \mathbb{S}^{N}\right) \times H_{A}$ is $H$-optimal for problem (55)-(56), (57) if

$$
\left(A^{0}, y^{0}\right) \in \Xi, \quad I\left(A^{0}, y^{0}\right)=\inf _{(A, y) \in \Xi} I(A, y) .
$$

Referring to Lions [24], we make use of the following assumption, which allows to establish a certain smoothness property for solutions of variational inequality (55).

Hypothesis 1. For a given set $K \subset W_{0}^{1,1}(\Omega)$, we have the following: for all $A \in \mathfrak{A}_{a d}$, for all $y \in K_{A}$, and for all $\varepsilon=\varepsilon(A)>0$ there exists an element $y_{\varepsilon} \in K_{A}$ such that

$$
\begin{gathered}
B_{A}\left(y_{\varepsilon}\right)=-\operatorname{div}\left(A \nabla y_{\varepsilon}\right)+y_{\varepsilon} \in L^{2}(\Omega), \\
y_{\varepsilon}+\varepsilon B_{A}\left(y_{\varepsilon}\right)=y .
\end{gathered}
$$

Then, as a direct consequence of Theorem 8.7 in [24] and Proposition 21, we come to the following result.

Theorem 22. Assume Hypothesis 1 holds true. Let $A \in \mathfrak{A}_{a d}$ be any admissible control and let $y=y(A)$ be the corresponding solution of variational inequality (55). Then $B_{A}(y) \in$ $L^{2}(\Omega)$ provided $f \in L^{2}(\Omega)$.

As an example of the set $K \in W_{0}^{1,1}(\Omega)$ satisfying the Hypothesis 1, we propose the following one.

Example 23. Let $\psi \in C_{0}^{\infty}(\Omega)$ be a given function such that

$$
-\operatorname{div}(A(x) \nabla \psi)+\psi \leq 0 \quad \forall A \in \mathfrak{A}_{a d} .
$$

Let us define the closed convex set $K$ as follows.

$$
K=\left\{v \mid v \in W_{0}^{1,1}(\Omega), v \geq \psi \text { a.e. in } \Omega\right\} .
$$

Then, for an arbitrary matrix $A \in \mathfrak{A}_{a d}$, we have

$$
\begin{aligned}
K_{A} & =c l\{K \cap H(\Omega ; A d x)\}_{H_{A}} \\
& =\{v \mid v \in H(\Omega ; A d x), \quad v \geq \psi \text { a.e. in } \Omega\} .
\end{aligned}
$$

Let us show that for all $y \in K_{A}$, for all $\varepsilon>0$ there exists an element $y_{\varepsilon} \in K_{A}$ satisfying the following conditions:

$$
-\operatorname{div}\left(A \nabla y_{\varepsilon}\right)+y_{\varepsilon} \in L^{2}(\Omega), \quad y_{\varepsilon}+\varepsilon B_{A}\left(y_{\varepsilon}\right)=y .
$$

As a result, we would have

$$
B_{A}\left(y_{\varepsilon}\right)-\frac{y-y_{\varepsilon}}{\varepsilon}=0
$$

and having set $y_{\varepsilon}-y=w_{\varepsilon} \in L^{2}(\Omega)$, we come to the equation

$$
B_{A}\left(w_{\varepsilon}\right)+\frac{w_{\varepsilon}}{\varepsilon}=-B_{A}(y) .
$$

Obviously, the mapping $H(\Omega ; A d x) \ni w_{\varepsilon} \rightarrow B_{A}\left(w_{\varepsilon}\right)+$ $w_{\varepsilon} / \varepsilon$ is strictly monotone and coercive (see Proposition 21). Hence, there exists a unique solution $w_{\varepsilon}$ to (73). Moreover, in this case $B_{A}\left(y_{\varepsilon}\right)=\left(y-y_{\varepsilon}\right) / \varepsilon \in L^{2}(\Omega)$.

Let us show that $y_{\varepsilon} \in K_{A}$, namely, $y_{\varepsilon} \geq \psi$ almost everywhere in $\Omega$. In order to do this, we set $z_{\varepsilon}=\sup \{\psi-$ $\left.y_{\varepsilon}, 0\right\}$. Then it is enough to prove that $z_{\varepsilon}=0$. From (72) it follows that

$$
B_{A}\left(\psi-y_{\varepsilon}\right)-B_{A}(\psi)=\frac{y_{\varepsilon}-y}{\varepsilon} .
$$

Having noticed $\left\langle B_{A}\left(\psi-y_{\varepsilon}\right), z_{\varepsilon}\right\rangle_{H_{A}}=\left\langle B_{A}\left(z_{\varepsilon}\right), z_{\varepsilon}\right\rangle_{H_{A}} \geq 0$, we conclude that

$$
\left\langle-B_{A}(\psi), z_{\varepsilon}\right\rangle_{H_{A}} \leq\left\langle\frac{y_{\varepsilon}-y}{\varepsilon}, z_{\varepsilon}\right\rangle_{H_{A}} .
$$

Since $z_{\varepsilon} \geq 0$ and $B_{A}(\psi) \leq 0$ by the initial assumptions, we have $\left\langle\left(y_{\varepsilon}-y\right) / \varepsilon, z_{\varepsilon}\right\rangle_{H_{A}} \geq 0$; that is,

$$
\begin{gathered}
\int_{E} \frac{y_{\varepsilon}-y}{\varepsilon} z_{\varepsilon} d x \geq 0, \\
E=\left\{x \mid x \in \Omega, \psi(x) \geq y_{\varepsilon}(x)\right\} .
\end{gathered}
$$

At the same time, since $y \in K_{A}$, then $y \geq \psi$ and, hence, $y \geq$ $y_{\varepsilon}$ on $E$. As a result, we have $y_{\varepsilon}-y \leq 0$ on $E$. Therefore,

$$
\int_{E} \frac{y_{\varepsilon}-y}{\varepsilon} z_{\varepsilon} d x \leq 0 \Longrightarrow \int_{E} \frac{y_{\varepsilon}-y}{\varepsilon} z_{\varepsilon} d x=0 .
$$

Thus, we arrive at the following conclusion: either $y_{\varepsilon}=$ $y$ or $z_{\varepsilon}=0$ a.e. on $E$. Hence, $z_{\varepsilon}=0$ and this concludes the proof. 


\section{Existence of Optimal Solutions}

Since our prime interest is the solvability of optimal control problem (55)-(56), (57), we begin with the study of the topological properties of the set of admissible solutions $\Xi$. To do so, we give some auxiliary results.

Definition 24. We say that a sequence $\left\{\left(A_{n}, y_{n}\right) \in \Xi\right\}_{n \in \mathbb{N}}$ is bounded if

$$
\sup _{n \in \mathbb{N}}\left[\left\|A_{n}\right\|_{B V\left(\Omega ; \mathbb{S}^{N}\right)}+\left\|y_{n}\right\|_{A_{n}}\right]<+\infty .
$$

Lemma 25. Let $\left\{\left(A_{n}, y_{n}\right) \in \Xi\right\}_{n \in \mathbb{N}}$ be a bounded sequence in the sense of Definition 24. Then there exists a pair $(A, y) \in$ $L^{1}\left(\Omega ; \mathbb{S}^{N}\right) \times W_{0}^{1,1}(\Omega)$ such that, up to a subsequence,

$$
\left(A_{n}, y_{n}\right) \stackrel{w}{\longrightarrow}(A, y), \quad A \in \mathfrak{A}_{a d}, \quad y \in H(\Omega ; A d x) .
$$

Proof. By the compactness of $B V$-functions (see Proposition 6), there exist a subsequence of $\left\{A_{n}\right\}_{n \in \mathbb{N}}$, still denoted by the same indices, and a matrix $A \in B V\left(\Omega ; \mathbb{S}^{N}\right)$ such that $A_{n} \rightarrow A$ in $L^{1}\left(\Omega ; \mathbb{S}^{N}\right)$. Thus,

$$
\begin{gathered}
A \in B V\left(\Omega ; \mathbb{S}^{N}\right), \\
\int_{\Omega} A(x) d x=\lim _{n \rightarrow \infty} \int_{\Omega} A_{n}(x) d x=M,
\end{gathered}
$$

and the condition (32) of Definition 14 holds true. In order to check the remaining conditions (33)-(34) of this definition and to show that $A \in \mathfrak{A}_{a d}$, we make use of the following observation.

We have $\left(A_{n}, y_{n}\right) \in \Xi$ for all $n \in \mathbb{N}$. Hence, there is a sequence $\left\{\zeta_{*, n}\right\}_{n \in \mathbb{N}}$ in $\Psi_{*}$ such that (see Lemma 13 for the details) $\zeta_{*, n} \rightarrow \zeta_{*}$ and $\zeta_{*, n}^{-1} \rightarrow \zeta_{*}^{-1}$ in $L^{1}(\Omega)$ as $n \rightarrow \infty$. Moreover, by properties of the set $\Psi_{*}$, the $L^{1}$-limit element $\zeta_{*}$ satisfies (7). Then, in view of $L^{1}$-convergence $A_{n} \rightarrow A$, we may assume that $A_{n}^{-1} \rightarrow A^{-1}$ almost everywhere in $\Omega$. Since $A_{n}(x) \geq \zeta_{*, n} I$ a.e. in $\Omega$, it follows that

$$
\int_{S}\left(\vec{\xi}, A_{n}^{-1} \vec{\xi}\right)_{\mathbb{R}^{N}} d x \leq \int_{S} \zeta_{*, n}^{-1} d x\|\vec{\xi}\|_{\mathbb{R}^{N}}^{2} \quad \forall n \in \mathbb{N}
$$

for any subset $S \subset \Omega$. Hence, due to the strong $L^{1}$ convergence $\zeta_{*, n}^{-1} \rightarrow \zeta_{*}^{-1}$, the sequence $\left\{A_{n}^{-1}\right\}_{n \in \mathbb{N}}$ is equiintegrable. Then, by Lebesgue's Theorem (see Lemma 2 ) we obtain $A_{n}^{-1} \rightarrow A^{-1}$ in $L^{1}\left(\Omega ; \mathbb{S}^{N}\right)$ as $n \rightarrow \infty$. As a result,

$$
A \in \mathfrak{M}_{\alpha}^{\beta}(\Omega) \cap L^{1}\left(\Omega ; \mathbb{S}^{N}\right)
$$

by Lemma 15 . Combining this fact with properties (80), we conclude $A \in \mathfrak{A}_{\text {ad }}$.

It remains to notice that conditions (33)-(34) of Definition 14 and $y \in H_{A}$ for the $w$-limiting component $(A, y)$ of the sequence $\left\{\left(A_{n}, y_{n}\right)\right\}_{n \in \mathbb{N}}$ are ensured by Lemma 15. This concludes the proof.

Our next step deals with the study of topological properties of the set of admissible solutions $\Xi$ to problem (55)-(56), (57). The following theorem is crucial for our next analysis.
Theorem 26. For any admissible initial data $f \in L^{2}(\Omega)$, the set of admissible solutions $\Xi$ is sequentially closed with respect to $w$-convergence.

Proof. Let $\left\{\left(A_{n}, y_{n}\right) \in \Xi\right\}_{n \in \mathbb{N}}$ be a bounded $w$-convergent sequence of admissible solutions to optimal control problem (55)-(56), (57). Let $(A, y)$ be its $w$-limit. Our aim is to prove that $(A, y) \in \Xi$. By Lemma 25, we have $A \in \mathfrak{A}_{a d}$ and $y \in$ $H(\Omega ; A d x)$.

Further, let us show that $y \in K_{A}$. Indeed, the condition $y_{n} \in K_{A_{n}}$ implies $y_{n} \in K$ for all $n \in \mathbb{N}$, whereas relations (33)(34) imply the weak convergence of $\left\{y_{n}\right\}_{n \in \mathbb{N}}$ to $y$ in $W_{0}^{1,1}(\Omega)$ within a subsequence (see (13)-(14)). Since the set $K$ is closed in $W_{0}^{1,1}(\Omega)$ and convex, then, in view of Mazur's lemma, $K$ is weakly closed in $W_{0}^{1,1}(\Omega)$. Therefore, $y \in K$ and $y \in$ $H(\Omega ; A d x)$. Combining these facts, we conclude that $y \in K_{A}$.

It remains to show that the pair $(A, y)$ satisfies relation (55) for all $v \in K_{A}$. In order to do this, we divide the rest of the proof into three steps.

Step 1. Let us show that for every $v \in K_{A}$, there exists a sequence $\left\{v_{n} \in K_{A_{n}}\right\}_{n \in \mathbb{N}}$, such that

$$
\begin{gathered}
v_{n} \longrightarrow v \quad \text { in } L^{2}(\Omega) \\
\nabla v_{n} \rightarrow \nabla v \text { in variable space } L^{2}\left(\Omega ; A_{n} d x\right)^{N} .
\end{gathered}
$$

Indeed, since $v \in H(\Omega ; A d x)$, it follows that there exists a sequence $\left\{\varphi_{n}\right\}_{n \in \mathbb{N}}$ in $C_{0}^{\infty}(\Omega)$ such that

$$
\begin{gathered}
\varphi_{n} \longrightarrow v \quad \text { in } L^{2}(\Omega), \\
\nabla \varphi_{n} \longrightarrow \nabla v \quad \text { in } L^{2}(\Omega ; A d x)^{N} \\
\text { as } n \longrightarrow \infty .
\end{gathered}
$$

Moreover, by the initial assumptions, $K_{A}$ contains only smooth functions and their limits with respect to the strong convergence in $H(\Omega ; A d x)$. Hence, $\varphi_{n} \in K_{A}$ for all indices large enough. Let us show that $\left\{\nabla \varphi_{n}\right\}_{n \in \mathbb{N}}$ converges to $\nabla v$ weakly in $L^{2}\left(\Omega ; A_{n} d x\right)^{N}$. To begin with, we show the boundedness of the sequence $\left\{\nabla \varphi_{n}\right\}_{n \in \mathbb{N}}$ in $L\left(\Omega ; A_{n} d x\right)^{N}$. Indeed,

$$
\begin{aligned}
& \limsup _{n \rightarrow \infty} \int_{\Omega}\left(A_{n} \nabla \varphi_{n}, \nabla \varphi_{n}\right)_{\mathbb{R}^{N}} d x \\
& \quad \leq \limsup _{n \rightarrow \infty} \int_{\Omega} \beta(x)\left\|\nabla \varphi_{n}(x)\right\|_{\mathbb{R}^{N}}^{2} d x \\
& \quad \leq \limsup _{n \rightarrow \infty} \int_{\Omega} \beta(x) \zeta_{*, n}^{-1}(x)\left(A \nabla \varphi_{n}, \nabla \varphi_{n}\right)_{\mathbb{R}^{N}} d x \\
& \quad<\limsup _{n \rightarrow \infty} \int_{\Omega} \beta(x) \zeta_{a d}^{-1}(x)\left(A \nabla \varphi_{n}, \nabla \varphi_{n}\right)_{\mathbb{R}^{N}} d x \\
& \quad \leq\left\|\beta \zeta_{a d}^{-1}\right\|_{L^{\infty}(\Omega)} \limsup _{n \rightarrow \infty} \int_{\Omega}\left(A \nabla \varphi_{n}, \nabla \varphi_{n}\right)_{\mathbb{R}^{N}} d x<\infty .
\end{aligned}
$$

In view of Proposition 9, the sequence $\left\{\nabla \varphi_{n}\right\}_{n \in \mathbb{N}}$, within a subsequence, is weakly convergent in the variable space 
$L\left(\Omega ; A_{n} d x\right)^{N}$. Let us show that $\nabla v$ is its weak limit. Let $\psi \in$ $C_{0}^{\infty}(\Omega)^{N}$ be an arbitrary smooth function. Then

$$
\begin{aligned}
& \left|\int_{\Omega}\left(A_{n} \nabla \varphi_{n}, \psi\right)_{\mathbb{R}^{N}} d x-\int_{\Omega}(A \nabla v, \psi)_{\mathbb{R}^{N}} d x\right| \\
& \leq\left|\int_{\Omega}\left(\left(A_{n}-A\right) \nabla \varphi_{n}, \psi\right)_{\mathbb{R}^{N}} d x\right| \\
& \quad+\left|\int_{\Omega}\left(A\left(\nabla \varphi_{n}-\nabla v\right), \psi\right)_{\mathbb{R}^{N}} d x\right| \\
& =I_{1}+I_{2} .
\end{aligned}
$$

Obviously, $I_{2} \rightarrow 0$ as $n \rightarrow \infty$ provided by the strong convergence $\varphi_{n} \rightarrow \quad v$ in $H(\Omega ; A d x)$. Further, using the Cauchy-Bunjakowsky inequality, we have

$$
\begin{aligned}
\left|\int_{\Omega}\left(\left(A_{n}-A\right) \nabla \varphi_{n}, \psi\right)_{\mathbb{R}^{N}} d x\right| \\
\leq\left(\int_{\Omega}\left(\left(A_{n}-A\right) \nabla \varphi_{n}, \nabla \varphi_{n}\right)_{\mathbb{R}^{N}} d x\right)^{1 / 2} \\
\quad \cdot\left(\int_{\Omega}\left(\left(A_{n}-A\right) \nabla \psi, \nabla \psi\right)_{\mathbb{R}^{N}} d x\right)^{1 / 2} \longrightarrow 0 \\
\text { as } n \rightarrow \infty
\end{aligned}
$$

as the product of a bounded sequence and a sequence converging to zero. It follows that

$$
\begin{array}{r}
\varphi_{n} \longrightarrow v \quad \text { strongly in } L^{2}(\Omega), \\
\nabla \varphi_{n} \rightarrow \nabla v \quad \text { weakly in } L^{2}\left(\Omega ; A_{n} d x\right) \\
\text { as } n \longrightarrow \infty .
\end{array}
$$

Step 2. Here we have to substantiate the correctness of the limit passage in the following variational inequality:

$$
\begin{array}{r}
\left\langle-\operatorname{div}\left(A_{n}(x) \nabla y_{n}\right)+y_{n}, v_{n}-y_{n}\right\rangle_{H_{A_{n}}} \geq\left\langle f, v_{n}-y_{n}\right\rangle_{H_{A_{n}}} \\
\forall v_{n} \in K_{A_{n}},
\end{array}
$$

where $y_{n} \in K_{A_{n}}$ is its solution. To do so, it is enough to show that

$$
\begin{array}{r}
\operatorname{div}\left(A_{n}(x) \nabla y_{n}\right) \rightarrow \operatorname{div}(A(x) \nabla y) \quad \text { in } L^{2}(\Omega) \\
\text { as } n \longrightarrow \infty .
\end{array}
$$

Since for every $n \in \mathbb{N}$ relation (89) holds true for every $v_{n} \in K_{A_{n}}$, we may consider only those elements $\left\{v_{n} \in\right.$ $\left.K_{A_{n}}\right\}_{n \in \mathbb{N}}$ which form convergent sequences to elements $v \in$ $K_{A}$ in a way that was established in Step 1. obtain

Taking into account Hypothesis 1 and Theorem 22, we

$$
\operatorname{div}\left(A_{n}(x) \nabla y_{n}\right) \in L^{2}(\Omega) \quad \forall n \in \mathbb{N},
$$

and, moreover, for an arbitrary $\psi \in C_{0}^{\infty}(\Omega)$, we have

$$
\begin{array}{r}
\left\langle\operatorname{div}\left(A_{n}(x) \nabla y_{n}\right), \psi\right\rangle_{L^{2}(\Omega)}=\int_{\Omega}\left(A_{n} \nabla y_{n}, \nabla \psi\right)_{\mathbb{R}^{N}} d x \\
\longrightarrow \int_{\Omega}(A \nabla y, \nabla \psi)_{\mathbb{R}^{N}} d x=\langle\operatorname{div}(A(x) \nabla y), \psi\rangle_{L^{2}(\Omega)} \\
\text { as } n \longrightarrow \infty
\end{array}
$$

Hence, the assertion (90) is valid.

Step 3. In view of the initial assumptions, we have

(1) $A_{n} \rightarrow A$ strongly in $L^{1}\left(\Omega ; \mathbb{S}^{N}\right)$;

(2) $y_{n} \rightarrow y$ in $L^{2}(\Omega)$;

(3) $\nabla y_{n} \rightarrow \nabla y$ in $L^{2}\left(\Omega ; A_{n} d x\right)$;

(4) $\operatorname{div}\left(A_{n}(x) \nabla y_{n}\right) \rightarrow \operatorname{div}(A(x) \nabla y)$ in $L^{2}(\Omega)$;

(5) for all $v \in K_{A}$ there exists a sequence $\left\{v_{n} \in K_{A_{n}}\right\}_{n \in \mathbb{N}}$, such that $v_{n} \rightarrow v$ strongly in $L^{2}(\Omega)$ and $\nabla v_{n} \rightarrow \nabla v$ in $L^{2}\left(\Omega ; A_{n} d x\right)$.

Hence, using this, Proposition 10 and the lower semicontinuity property of $L^{2}$-norm with respect to the weak convergence, we obtain

$$
\begin{aligned}
\langle-\operatorname{div} & (A(x) \nabla y)+y, v-y\rangle_{H_{A}} \\
= & \langle-\operatorname{div}(A(x) \nabla y)+y, v\rangle_{L^{2}(\Omega)} \\
& -\int_{\Omega}\left((A \nabla y, \nabla y)_{\mathbb{R}^{N}}+y^{2}\right) d x \\
\geq & \limsup _{n \rightarrow \infty}\left\langle-\operatorname{div}\left(A_{n}(x) \nabla y_{n}\right)+y_{n}, v_{n}\right\rangle_{L^{2}(\Omega)} \\
& -\liminf _{n \rightarrow \infty} \int_{\Omega}\left(\left(A_{n} \nabla y_{n}, \nabla y_{n}\right)_{\mathbb{R}^{N}}+y_{n}^{2}\right) d x \\
\geq & \limsup _{n \rightarrow \infty}\left(\left\langle-\operatorname{div}\left(A_{n}(x) \nabla y_{n}\right)+y_{n}, v_{n}\right\rangle_{H_{A_{n}}}\right. \\
& \left.\quad-\int_{\Omega}\left(\left(A_{n} \nabla y_{n}, \nabla y_{n}\right)_{\mathbb{R}^{N}}+y_{n}^{2}\right) d x\right) \\
\geq & \lim _{n \rightarrow \infty}\left\langle f, v_{n}-y_{n}\right\rangle_{H_{A_{n}}}=\lim _{n \rightarrow \infty}\left\langle f, v_{n}-y_{n}\right\rangle_{L^{2}(\Omega)} \\
= & \langle f, v-y\rangle_{H_{A}} \forall v \in K_{A} .
\end{aligned}
$$

As a result, we come to the desired relation:

$$
\langle-\operatorname{div}(A(x) \nabla y)+y, v-y\rangle_{H_{A}} \geq\langle f, v-y\rangle_{H_{A}} \quad \forall v \in K_{A},
$$

which means that the limit pair $(A, y)$ belongs to the set of admissible solutions of problem (55)-(56), (57). Hence, set $\Xi$ is sequentially closed with respect to $w$-convergence in $L^{1}\left(\Omega ; \mathbb{S}^{N}\right) \times H\left(\Omega ; A_{n} d x\right)$, and this concludes the proof.

We are now in a position to state the existence of $H$ optimal solution to problem (55)-(56), (57). 
Theorem 27. Let $f \in L^{2}(\Omega)$ and $y_{d} \in L^{2}(\Omega)$ be given functions. Assume that the Hypothesis 1 is valid. Then optimal control problem (55)-(56), (57) admits at least one solution $\left(A^{0}, y^{0}\right) \in L^{1}\left(\Omega ; \mathbb{S}^{N}\right) \times H\left(\Omega ; A_{0} d x\right)$.

Proof. Since the cost functional $I=I(A, y)$ is bounded below and $\Xi \neq \emptyset$, it provides the existence of a minimizing sequence $\left\{\left(A_{n}, y_{n}\right) \in \Xi\right\}_{n \in \mathbb{N}}$ to the problem (66). Then,

$$
\begin{aligned}
\inf _{(A, y) \in \Xi} I(A, y)=\lim _{n \rightarrow \infty} I & \left(A_{n}, y_{n}\right) \\
=\lim _{n \rightarrow \infty} & {\left[\int_{\Omega}\left|y_{n}(x)-y_{d}(x)\right|^{2} d x\right.} \\
& +\int_{\Omega}\left(\nabla y_{n}(x), A(x) \nabla y_{n}(x)\right)_{\mathbb{R}^{N}} d x \\
& \left.+\sum_{i, j=1}^{N} \int_{\Omega \backslash Q}\left|D a_{i j}^{n}(x)\right|\right]<+\infty
\end{aligned}
$$

implies the existence of a constant $C>0$ such that

$$
\begin{gathered}
\sup _{n \in \mathbb{N}}\left\|\nabla y_{n}\right\|_{L^{2}\left(\Omega, A_{n} d x\right)^{N}} \leq C, \\
\sup _{n \in \mathbb{N}}\left\|y_{n}\right\|_{L^{2}(\Omega)} \leq C, \\
\sup _{n \in \mathbb{N}}\left\|A_{n}\right\|_{B V\left(\Omega \backslash Q ; \mathbb{S}^{N}\right)} \leq C .
\end{gathered}
$$

Hence, the minimizing sequence $\left\{\left(A_{n}, y_{n}\right) \in \Xi\right\}_{n \in \mathbb{N}}$ is bounded in the sense of Definition 24. Then, by Lemma 25, there exist functions $A^{0} \in L^{1}\left(\Omega ; \mathbb{S}^{N}\right)$ and $y^{0} \in H\left(\Omega ; A_{0} d x\right)$ such that, up to a subsequence, $\left(A_{n}, y_{n}\right) \stackrel{w}{\rightarrow}\left(A^{0}, y^{0}\right)$. Since the set $\Xi$ is sequentially closed with respect to the $w$-convergence (see Theorem 26), it follows that the $w$-limit pair $\left(A^{0}, y^{0}\right)$ is an admissible solution to (55)-(56), (57) (i.e., $\left.\left(A^{0}, y^{0}\right) \in \Xi\right)$. To conclude the proof, it is enough to observe that the cost functional $I$ is sequentially lower $w$-semicontinuous. Hence,

$$
I\left(A^{0}, y^{0}\right) \leq \liminf _{n \rightarrow \infty} I\left(A_{n}, y_{n}\right)=\inf _{(A, y) \in \Xi} I(A, y)
$$

that is, $\left(A^{0}, y^{0}\right)$ is an optimal solution. The proof is complete.

\section{References}

[1] P. I. Kogut and G. Leugering, "Optimal $L^{1}$-control in coefficients for Dirichlet elliptic problems: $H$-optimal solutions," Zeitschrift für Analysis und ihre Anwendungen, vol. 31, no. 1, pp. 31-53, 2012.

[2] P. I. Kogut and G. Leugering, "Optimal $L^{1}$-control in coefficients for Dirichlet elliptic problems: $W$-optimal solutions," Journal of Optimization Theory and Applications, vol. 150, no. 2, pp. 205232, 2011.

[3] C. Calvo-Jurado and J. Casado-Díaz, "Optimization by the homogenization method for nonlinear elliptic Dirichlet systems," Mediterranean Journal of Mathematics, vol. 4, no. 1, pp. 53-63, 2007.
[4] G. Duvaut and J.-L. Lions, Inequalities in Mechanics and Physics, Springer, Berlin, Germany, 1976.

[5] D. Kinderlehrer and G. Stampacchia, An Introduction to Variational Inequalities and Their Applications, vol. 88, Academic Press, New York, NY, USA, 1980.

[6] U. Leonhardt, "Optical conformal mapping," American Association for the Advancement of Science, vol. 312, no. 5781, pp. 17771780, 2006.

[7] J. Pendry, "Negative refraction makes a perfect lens," Physical Review Letters, vol. 8, pp. 3966-3969, 2000.

[8] A. Greenleaf, Y. Kurylev, M. Lassas, and G. Uhlmann, "Cloaking devices, electromagnetic wormholes, and transformation optics," SIAM Review, vol. 51, no. 1, pp. 3-33, 2009.

[9] R. Dautray and J.-L. Lions, Mathematical Analysis and Numerical Methods for Science and Technology, vol. 1 of Physical Origins and Classical Methods, Springer, Berlin, Germany, 1985.

[10] J. Lü, X. Yu, G. Chen, and D. Cheng, "Characterizing the synchronizability of small-world dynamical networks," IEEE Transactions on Circuits and Systems I, vol. 51, no. 4, pp. 787796, 2004.

[11] J. Lü and G. Chen, "A time-varying complex dynamical network model and its controlled synchronization criteria," IEEE Transactions on Automatic Control, vol. 50, no. 6, pp. 841-846, 2005.

[12] J. Zhou, J.-a. Lu, and J. Lü, "Adaptive synchronization of an uncertain complex dynamical network," IEEE Transactions on Automatic Control, vol. 51, no. 4, pp. 652-656, 2006.

[13] P. Caldiroli and R. Musina, "On a variational degenerate elliptic problem," Nonlinear Differential Equations and Applications, vol. 7, no. 2, pp. 187-199, 2000.

[14] J. Chabrowski, "Degenerate elliptic equation involving a subcritical Sobolev exponent," Portugaliae Mathematica, vol. 53, no. 2, pp. 167-177, 1996.

[15] P. I. Kogut and G. R. Leugering, Optimal Control Problems for Partial Differential Equations on Reticulated Domains, Birkhäuser, Boston, Mass, USA, 2011.

[16] M. K. V. Murthy and V. Stampacchia, "Boundary problems for some degenerate elliptic operators," Annali di Matematica Pura ed Applicata, vol. 5, no. 4, pp. 1-122, 1968.

[17] S. E. Pastukhova, "Degenerate equations of monotone type: Lavrentev phenomenon and attainability problems," Sbornik: Mathematics, vol. 198, no. 10, pp. 1465-1494, 2007.

[18] V. V. Zhikov and S. E. Pastukhova, "Homogenization of degenerate elliptic equations," Sbornik: Mathematics, vol. 49, no. 1, pp. 101-124, 2008.

[19] G. Bouchitté and G. Buttazzo, "Characterization of optimal shapes and masses through Monge-Kantorovich equation," Journal of the European Mathematical Society, vol. 3, no. 2, pp. 139-168, 2001.

[20] G. Buttazzo and N. Varchon, "On the optimal reinforcement of an elastic membrane," Rivista di Matematica della Università di Parma, vol. 4, no. 7, pp. 115-125, 2005.

[21] G. Buttazzo and P. I. Kogut, "Weak optimal controls in coefficients for linear elliptic problems," Revista Matemática Complutense, vol. 24, no. 1, pp. 83-94, 2011.

[22] C. D’Apice, U. De Maio, and O. P. Kogut, "Optimal control problems in coefficients for degenerate equations of monotone type: shape stability and attainability problems," SIAM Journal on Control and Optimization, vol. 50, no. 3, pp. 1174-1199, 2012.

[23] O. P. Kupenko, "Optimal control in coefficients for degenerate variational inequalities of monotone type. I: existence of optimal solutions," Journal of Numerical and Applied Mathematics, vol. 106, no. 3, pp. 88-104, 2011. 
[24] J. L. Lions, Some Methods of Solving Non-Linear Boundary Value Problems, Dunod-Gauthier-Villars, Paris, France, 1969.

[25] M. Z. Zgurovsky, V. S. Mel'nik, and P. O. Kasyanov, Evolution inclusions and variation inequalities for earth data processing I, vol. 24, Springer, Berlin, Germany, 2011.

[26] O. P. Kogut, "On optimal control problem in coefficients for nonlinear elliptic variational inequalities," Bulletin of Dnipropetrovsk National University, vol. 19, no. 3, pp. 86-98, 2011.

[27] F. Murat, "Un contre-exemple pour le problème du contrôle dans les coefficients," vol. 273, pp. A708-A711, 1971.

[28] U. E. Raĭtum, Optimal Control Problems for Elliptic Equations, Zinatne, Riga, Latvia, 1989.

[29] P. I. Kogut and G. Leugering, "Matrix-valued $L^{1}$-optimal control in the coefficients of linear elliptic problems ," In press.

[30] V. Chiadò Piat and F. Serra Cassano, "Some remarks about the density of smooth functions in weighted Sobolev spaces," Journal of Convex Analysis, vol. 1, no. 2, pp. 135-142, 1994.

[31] V. V.Zhikov, "On weighted Sobolev spaces," Sbornik: Mathematics, vol. 189, no. 8, pp. 27-58, 1998.

[32] E. Giusti, Minimal Surfaces and Functions of Bounded Variation, vol. 80 of Monographs in Mathematics, Birkhäuser Verlag, Basel, Switzerland, 1984.

[33] V. V. Zhikov, "On an extension and an application of the twoscale convergence method," Sbornik: Mathematics, vol. 191, no. 7, pp. 31-72, 2000. 


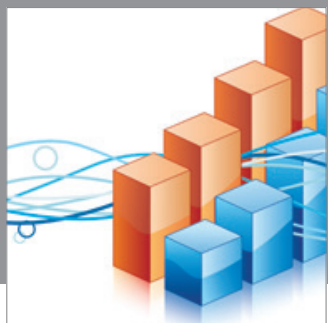

Advances in

Operations Research

mansans

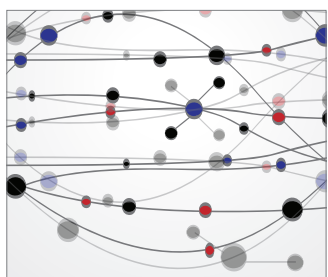

The Scientific World Journal
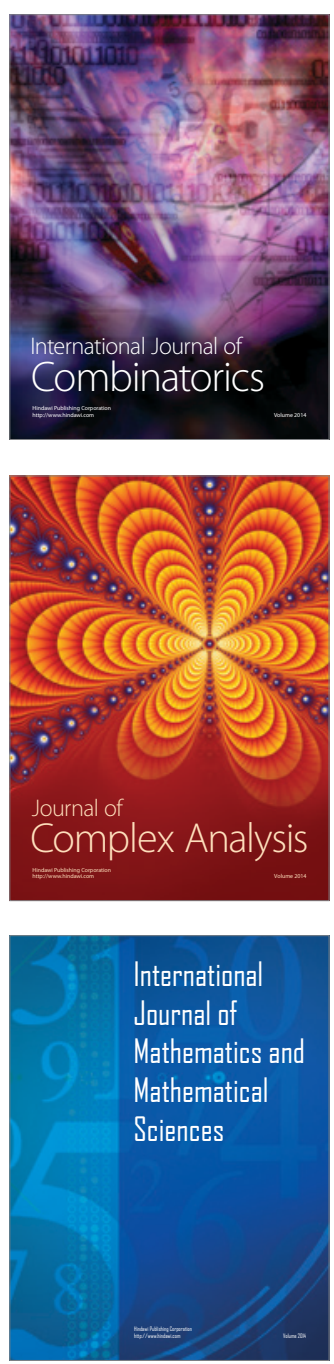
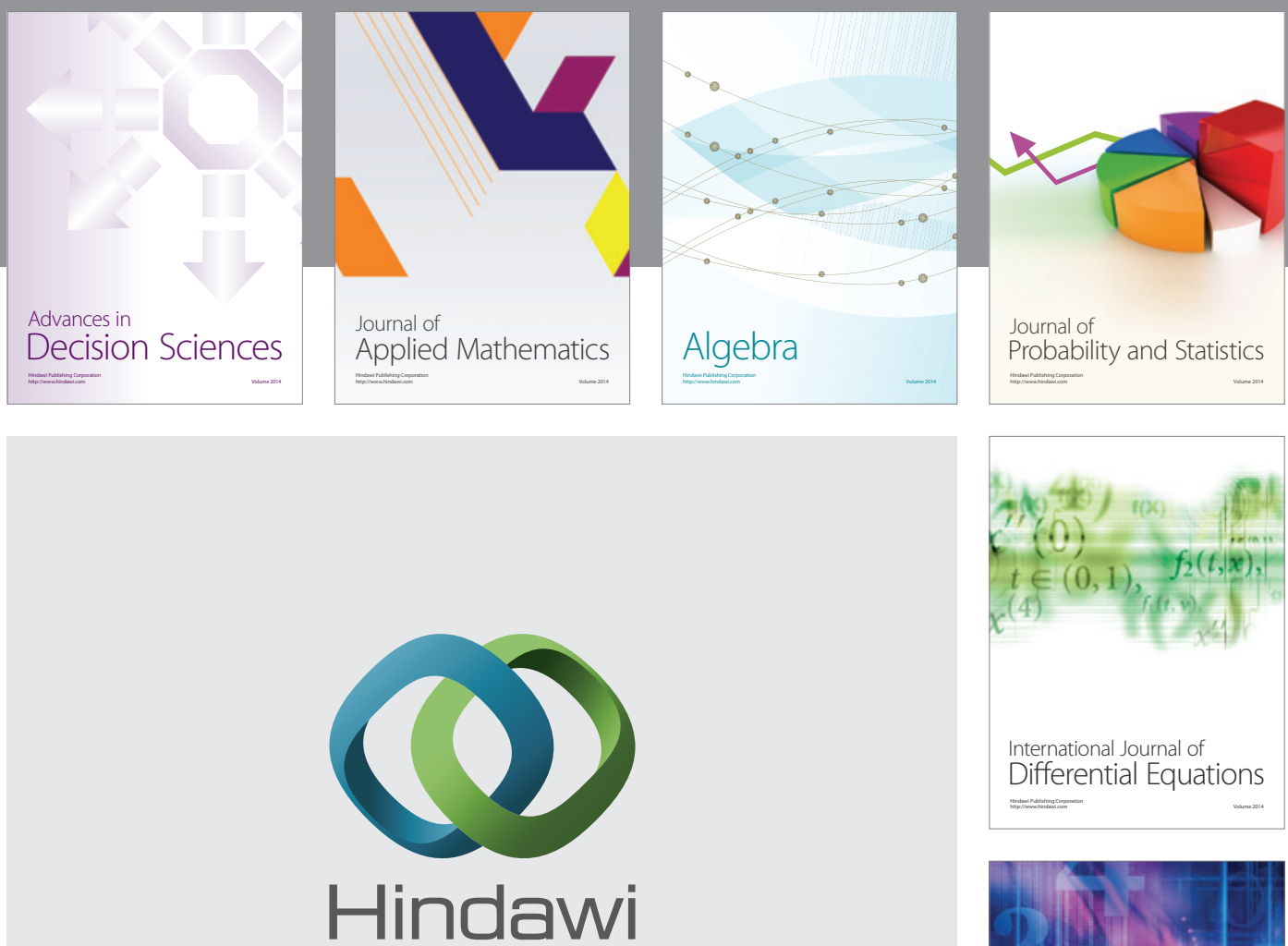

Submit your manuscripts at http://www.hindawi.com
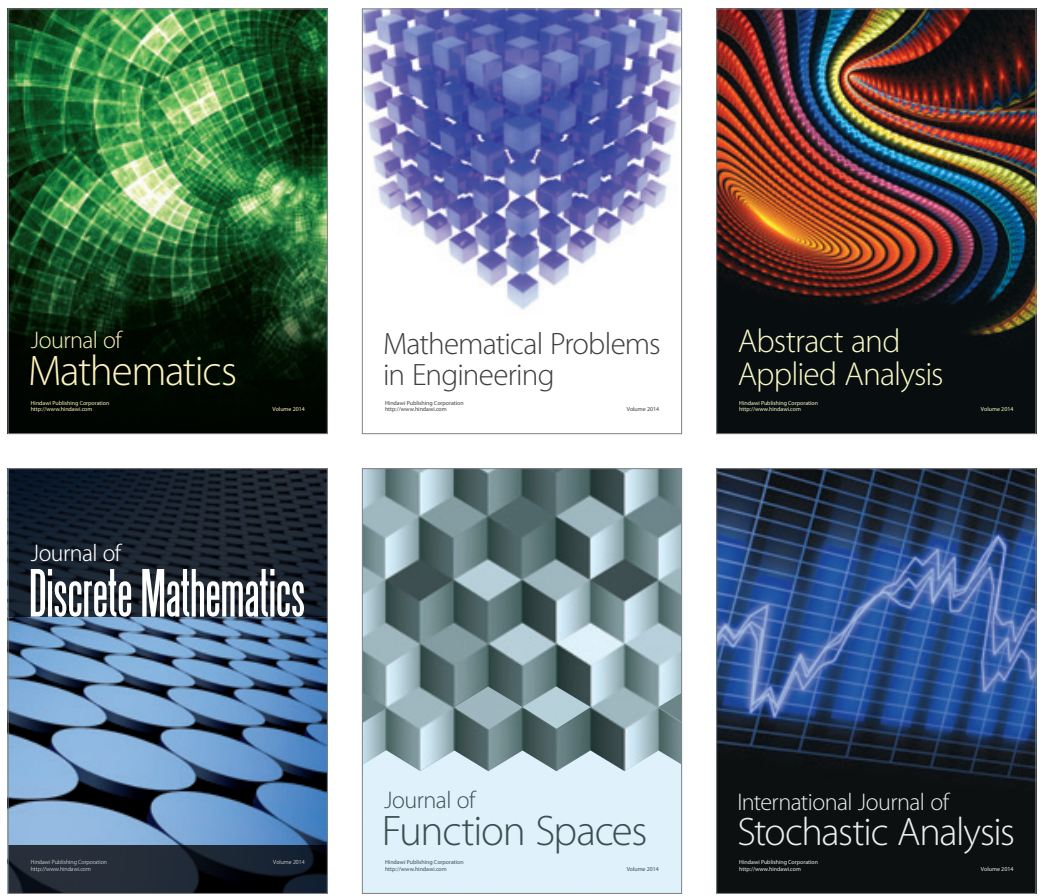

Journal of

Function Spaces

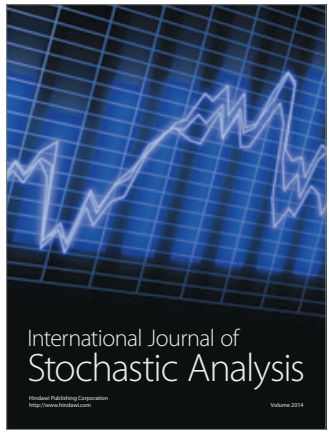

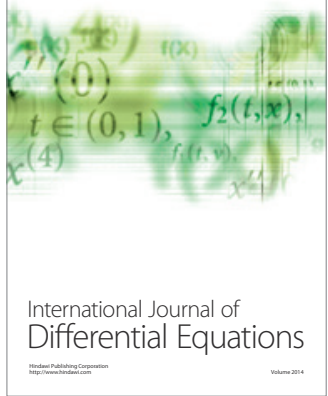
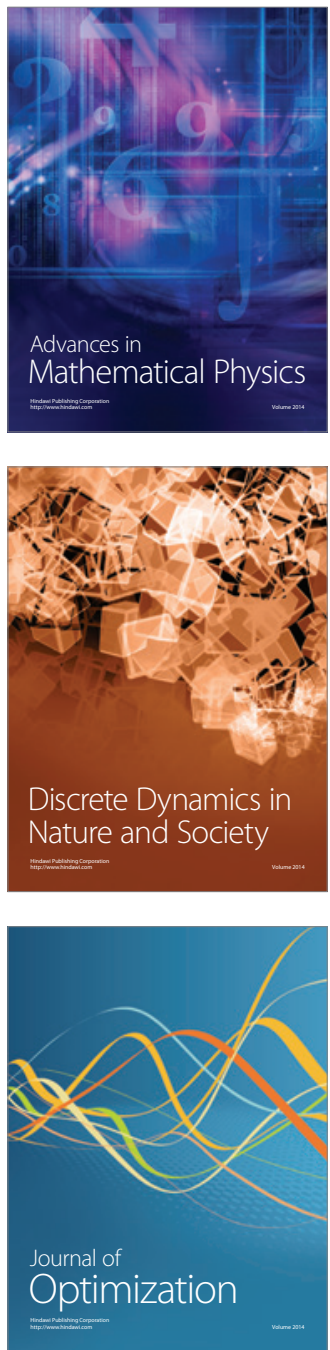